\title{
Targeting a proteolytic neoepitope on CUB domain containing protein 1 (CDCP1) for RAS-driven cancers
}

\author{
Shion A. Lim, ${ }^{1}$ Jie Zhou, ${ }^{1}$ Alexander J. Martinko, ${ }^{1}$ Yung-Hua Wang, ${ }^{2,3}$ Ekaterina V. Filippova, ${ }^{4,5}$ Veronica Steri, ${ }^{3,6}$ Donghui Wang, ${ }^{3,6}$ \\ Soumya C. Remesh, ${ }^{1}$ Jia Liu, ${ }^{1}$ Byron Hann, ${ }^{6}$ Anthony A. Kossiakoff,, ${ }^{4,5}$ Michael J. Evans, ${ }^{2,3}$ Kevin K. Leung, ${ }^{1}$ and James A. Wells ${ }^{17,8}$ \\ 'Department of Pharmaceutical Chemistry, ${ }^{2}$ Department of Radiology and Biomedical Imaging, and ${ }^{3}$ Helen Diller Family Comprehensive Cancer Center, UCSF, San Francisco, California, USA. ${ }^{4}$ Department of \\ Biochemistry and Molecular Biology, and ${ }^{5}$ Institute for Biophysical Dynamics, The University of Chicago, Chicago, Illinois, USA. ${ }^{6}$ Preclinical Therapeutics Core, UCSF, San Francisco, California, USA. \\ ${ }^{7}$ Chan Zuckerberg Biohub, San Francisco, California, USA. ${ }^{8}$ Department of Cellular and Molecular Pharmacology, UCSF, San Francisco, California, USA.
}

\begin{abstract}
Extracellular proteolysis is frequently dysregulated in disease and can generate proteoforms with unique neoepitopes not found in healthy tissue. Here, we demonstrate that Abs that selectively recognize a proteolytic neoepitope on CUB domain containing protein 1 (CDCP1) could enable more effective and safer treatments for solid tumors. CDCP1 is highly overexpressed in RAS-driven cancers, and its ectodomain is cleaved by extracellular proteases. Biochemical, biophysical, and structural characterization revealed that the 2 cleaved fragments of CDCP1 remain tightly associated with minimal proteolysis-induced conformational change. Using differential phage display, we generated recombinant Abs that are exquisitely selective to cleaved CDCP1 with no detectable binding to the uncleaved form. These Abs potently targeted cleaved CDCP1-expressing cancer cells as an Ab-drug conjugate, an Ab-radionuclide conjugate, and a bispecific T cell engager. In a syngeneic pancreatic tumor model, these cleaved-specific Abs showed tumor-specific localization and antitumor activity with superior safety profiles compared with a pan-CDCP1 approach. Targeting proteolytic neoepitopes could provide an orthogonal "AND" gate for improving the therapeutic index.
\end{abstract}

\section{Introduction}

A key to safe and effective cancer treatments is to selectively target diseased over healthy cells. Traditionally, this has been enabled by targeting antigens with large expression differences between diseased and normal tissue, but this severely limits the druggable target space. More recently, there has been interest in targeting disease-associated alternate splice forms or peptide-MHC complexes $(1,2)$. These are often rare conformations, and their low abundance has posed challenges. Proteolysis plays critical roles in both normal and aberrant biological processes (3) and is well known to be upregulated and/or dysregulated in a variety of disease, including cancer $(4,5)$. Recently, there have been efforts to better understand disease-associated proteases and their substrates, and therapeutic strategies to inhibit proteases or utilize them for conditional activation of masked therapeutics have been explored (6-9). Tumor-associated proteolysis may also produce novel epitopes on the surface of cancer cells. We hypothesize that therapeutics that recognize these proteolytic neoepitopes could address the challenge of on-target, off-tumor toxicity. However, generation of Abs that can specifically recog-

Authorship note: SAL and JZ are co-first authors and contributed equally to the study. Conflict of interest: SAL, JZ, AJM, JL, KKL, and JAW are inventors on a provisional patent (US provisional application no. 63/170,338) filed on the composition of matter for the antibodies described in this study.

Copyright: @ 2022, Lim et al. This is an open access article published under the terms of the Creative Commons Attribution 4.0 International License.

Submitted: August 30, 2021; Accepted: December 21, 2021; Published: February 15, 2022. Reference information: J Clin Invest. 2022;132(4):e154604.

https://doi.org/10.1172/JCl154604. nize proteolytic neoepitopes (10), particularly those on the cell surface, has not been demonstrated.

Ras activation is one of the most well-known oncogenic transformations and is implicated in many solid tumors, including in nearly $90 \%$ of pancreatic cancer occurrences (11). CUB domain containing protein 1 (CDCP1), also known as Trask, gp140, SIMA135, and CD318, is a type I single-pass membrane protein that is highly overexpressed in a variety of solid tumors (12-14). CDCP1 is upregulated and functionally critical in KRAS-transformed cells, and overexpression of CDCP1 is associated with loss of adhesion, aggressive metastasis, and poor prognosis (15-17). Full-length CDCP1 (fl-CDCP1) is a $135 \mathrm{kDa}$ protein composed of a large ectodomain, a transmembrane domain, and a short intracellular domain and is found on several types of tissue, including epithelial tissue along the gastrointestinal tract (18). The heavily glycosylated ectodomain of CDCP1 is predicted to contain 3 CUB-like domains, while the intracellular domain contains 5 tyrosine phosphorylation sites. Apart from this, the structure of CDCP1 has remained unknown.

Extracellular serine proteases have been shown to cleave CDCP1 into an N-terminal fragment (NTF) and a C-terminal fragment (CTF) (19). Proteolysis, along with overexpression, has been associated with the tumor-promoting functions of CDCP1, which involve phosphorylation of intracellular tyrosine residues and initiation of downstream signaling pathways associated with loss of adhesion, increased migration, and anoikis $(20,21)$. There is a higher proportion of cleaved CDCP1 (c-CDCP1) present in more aggressive metastatic cancer cell types compared with less malignant cancer cell lines (22). Given that protease levels and proteo- 
lytic activity are elevated in the tumor microenvironment (4), it is not surprising that $\mathrm{c}-\mathrm{CDCP} 1$ is more prevalent in aggressive cancers $(23,24)$ and that normal tissues almost exclusively express fl-CDCP1 (25-27). Thus, we hypothesized that therapeutic agents that can specifically target c-CDCP1 over fl-CDCP1 may expand the therapeutic window.

Here, we report the biochemical and biophysical characterization of $\mathrm{c}-\mathrm{CDCP} 1$ and the generation of recombinant Abs that specifically target this proteoform. We identified 3 cleavage sites of CDCP1 and found, surprisingly, that the NTF and CTF remained intact after proteolysis, with minimal structural rearrangement. The predicted structure of fl-CDCP1 by the recent AlphaFold (https://alphafold.ebi.ac.uk/) release corroborates our finding, revealing a large interface between NTF and CTF. Using a differential phage display strategy, we selected and optimized Abs that can specifically recognize an epitope on c-CDCP1 with no binding to uncleaved CDCP1. These Abs selectively target c-CDCP1-expressing pancreatic cancer cells and reduce tumor growth in vivo while substantially improving the safety profile compared with a pan-CDCP1-targeting approach. Ab-based targeting of proteolytic neoepitopes offers what we believe to be a novel strategy for expanding the therapeutic index.

\section{Results}

The NTF of CDCP1 is retained upon proteolysis. CDCP1 has been reported to be cleaved at the dibasic residues between the NTF and CTF (R368/K369; ref. 18). To characterize CDCP1 proteolysis and target c-CDCP1, we first generated recombinant proteins and cell lines expressing CDCP1 with an engineered cut site between the NTF and CTF, where we replaced R368/K369 with a PreScission Protease (Px) recognition sequence to inducibly control CDCP1 proteolysis (CDCP1[Px]) (Figure 1A). We additionally generated a variant where R368 and K369 were mutated to alanine (CDCP1[R368A/ $\mathrm{K} 369 \mathrm{~A}]-\mathrm{Fc}$ ) to prevent cleavage by basic residue-specific proteases and another where only the NTF (aa 30-369) was fused to an Fc domain (NTF-Fc). Efforts to recombinantly express the CTF (aa 370-665) alone were unsuccessful, suggesting NTF plays a role in expression and folding. Addition of Px cleaved CDCP1(Px)-Fc into 2 fragments (NTF and CTF) of expected molecular weight (Figure 1B). Surprisingly, we found that Px-treated CDCP1(Px)-Fc had the same size-exclusion chromatography (SEC) elution profile as uncleaved CDCP1-Fc, with no evidence of an unbound NTF (Figure 1C). Additionally, we observed robust binding by biolayer interferometry (BLI) of IgG 4A06, an Ab that recognizes the NTF of CDCP1 (Figure 1D and Supplemental Figure 1). To determine whether this was unique to Px cleavage, we generated and tested a thrombin protease-cleavable CDCP1-Fc (CDCP1[Tx]-Fc) (Supplemental Figure 2) and observed the same phenomena. To determine whether c-CDCP1 remains a complex on the cell membrane, we engineered cell lines expressing the fl-CDCP1 protein sequence with an N-terminal FLAG-tag and the R368/K369 proteolysis site replaced with the Px recognition sequence (Figure $1 \mathrm{E}$ ). Using an anti-CDCP1 Ab (D1W9N) that recognizes the intracellular C-terminal region of CDCP1, we observed that, although addition of Px to cells cleaves $\mathrm{CDCP} 1(\mathrm{Px})$ at the expected molecular weight, staining by antiFLAG and IgG 4A06 was unaffected, indicating that the NTF remains membrane associated after proteolysis (Figure $1 \mathrm{~F}$ ).
$I P-M S$ reveals 3 major cleavage sites for CDCP1. Confirming the precise proteolysis site(s) of CDCP1 on cancer cells is critical to designing the appropriate antigen for Ab generation. We used IP mass spectrometry (IP-MS) on a panel of pancreatic ductal adenocarcinoma (PDAC) cell lines expressing differential amounts of fl-CDCP1 and c-CDCP1 to map the proteolysis site(s) (Figure 2A). HPAC primarily expresses fl-CDCP1, whereas PL5 and PL45 express C-CDCP1, and HPNE is a nonmalignant pancreatic cell line with no CDCP1 expression (Figure 2B). Samples were treated with Glu-C to preserve the basic cut site(s) of c-CDCP1. We identified 3 unique cut sites after basic residues between the NTF and CTF; these included proteolysis after K365 (cut 1), R368 (cut 2), and $\mathrm{K} 369$ (cut 3). Cut 2 and cut 3 are previously reported proteolysis sites (28), while cut 1 appears to be a novel site (Figure 2C and Supplemental Figure 3). Peptides corresponding to the c-CDCP1 sequence were only observed in PL5 and PL45 cells, while only peptides mapping to the uncleaved sequence were found in HPAC and no CDCP1 peptides were observed for HPNE. These findings confirm that endogenous c-CDCP1 remains as a complex on PDAC cells and that CDCP1 is proteolytically processed between CUB1 and CUB2 to produce a heterogenous set of cleaved forms.

Cleaved and uncleaved CDCP1 adopt similar conformations. We reasoned that because c-CDCP1 forms a tight NTF/CTF complex, we could generate recombinant c-CDCP1 with the native cut sites by cotransfection of the 2 fragments. Cotransfection of one set of plasmids encoding the NTF of c-CDCP1 with a second set of plasmids encoding the CTF ectodomain of c-CDCP1 resulted in an intact c-CDCP1 complex for all 3 cut variants (c-CDCP1 cut 1, cut 2, cut 3; Figure 3, A and B, and Supplemental Figure 4, A-D). fl-CDCP1 and c-CDCP1 both bound robustly to IgG 4A06 (Figure 3C) and had similar SEC profiles (Supplemental Figure 4E), demonstrating that c-CDCP1 forms a complex even when generated in trans. Remarkably, c-CDCP1 and fl-CDCP1 ectodomains have similar melting temperatures (Figure 3D), indicating that the NTF/CTF complex is stable and does not dissociate until unfolding of the entire ectodomain.

We examined the structure of fl-CDCP1 and c-CDCP1 ectodomains by circular dichroism (CD) spectroscopy (Figure 3E), SEC-small-angle X-ray scattering (SEC-SAXS) (Figure 3, F and G, and Supplemental Figure 5), and SEC-multi-angle light scattering (SEC-MALS) (Figure $3 \mathrm{H}$ ). The CD spectra of fl-CDCP1 and $\mathrm{c}-\mathrm{CDCP} 1$ indicated a classic $\beta$ sheet signal, consistent with the CUB domain fold. There was a noticeable change in the spectral shape and minima between fl-CDCP1 and c-CDCP1, which suggests that proteolysis may cause subtle changes in the secondary structure of CDCP1. Comparison of the SEC-SAXS pair distance distribution functions and radii of gyration shows that both fl-CDCP1 and c-CDCP1 exhibit similar overall domain arrangement with no large-scale conformational changes as a result of proteolysis (Figure 3, F and G, and Supplemental Figure 5B). Furthermore, SEC-MALS showed that fl-CDCP1 and c-CDCP1 had the same elution profile and molecular weight ( 97-99 kDa), consistent with the predicted size of a monomeric ectodomain (77 kDa plus glycosylation) (Figure $3 \mathrm{H}$ and Supplemental Table 1). Overall, these data show that, other than small differences in the $\beta$ sheet signature, the conformation of fl-CDCP1 and c-CDCP1 are remarkably similar. 
A

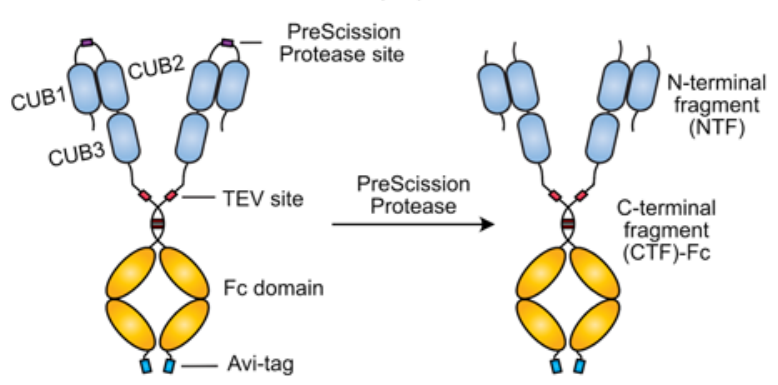

D
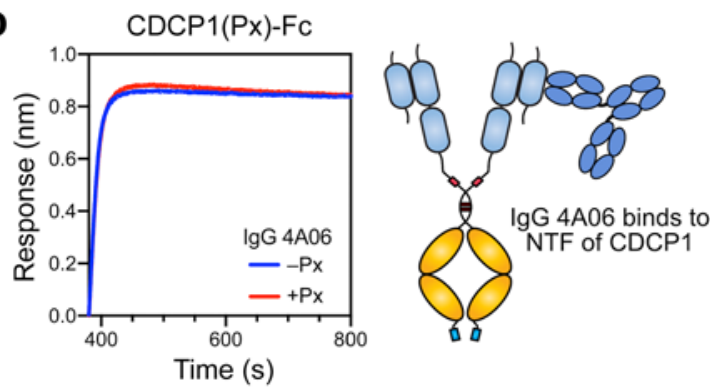

B

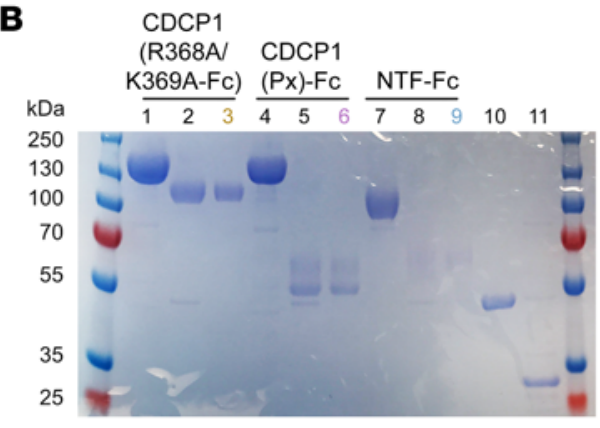

PreScission - + + + + + + +

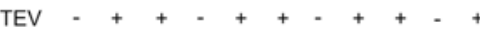

C

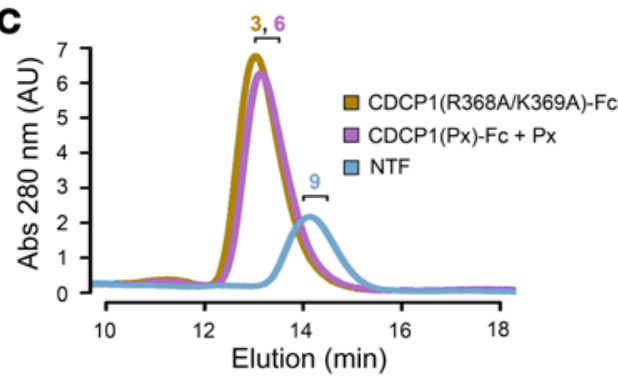

E HEK293T-CDCP1(Px)

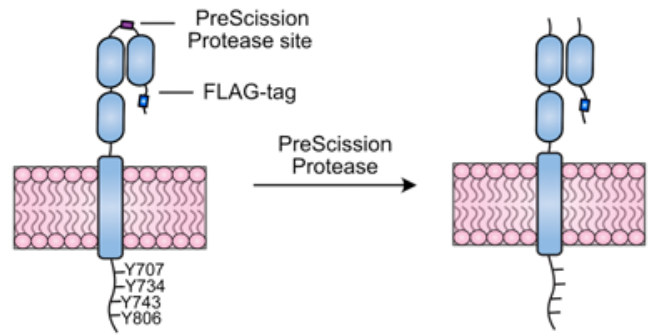

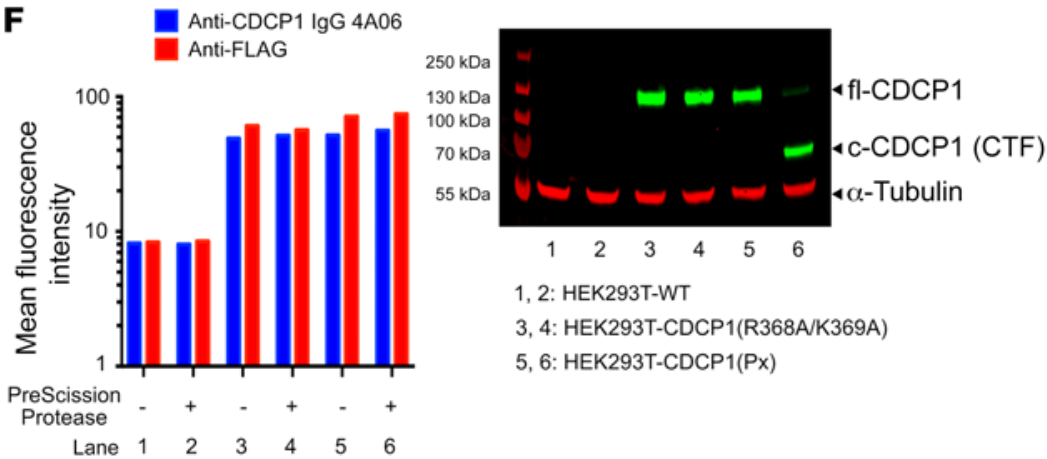

Figure 1. NTF of CDCP1 is retained upon proteolysis between the CUB1 and CUB2 domains. (A) Design of a Px-cleavable CDCP1 ectodomain fused to a TEV-releasable Fc domain with C-terminal avi-tag (CDCP1[Px]-Fc). The R368/K369 cleavage site was replaced with Px recognition sequence (CS) ${ }_{5}-$ LEVLFQGP-(CS) . (B) SDS-PAGE of CDCP1 constructs. Px treatment cleaves CDCP1(Px)-Fc into NTF and CTF-Fc fragments. NTF is heavily glycosylated (predicted $14 \mathrm{~N}$-linked glycosylation sites) and runs as a smeared higher-molecular weight band at approximately $60 \mathrm{kDa}$. (C) SEC of CDCP1(R368/K369A)Fc and CDCP1(Px)-Fc treated with Px and NTF (TEV released) shows that the NTF and CTF of CDCP1(Px) remain intact after proteolysis. Numbers denote fractions corresponding to the SDS-PAGE gel lanes in B. (D) BLI of IgG 4A06, which recognizes the NTF, shows robust binding to both Px-treated and untreated CDCP1(Px)-Fc. (E) Design of Px-cleavable CDCP1 with N-terminal FLAG-tag expressed in HEK293T cells. (F) Flow cytometry and Western blot of HEK293T-WT, HEK293T-CDCP1(R368A/K369A), and HEK293T-CDCP1(Px). Flow cytometry signal of anti-FLAC and IgC 4A06 remains unchanged with Px treatment. Western blot with anti-CDCP1 D1W9N, which recognizes the C-terminal intracellular region of CDCP1, shows Px-mediated CDCP1 proteolysis at the intended molecular weight.

Recently, DeepMind released AlphaFold, which provides high-confidence structural predictions of virtually the entire human proteome (29). The AlphaFold prediction of the CDCP1 structure is remarkably consistent with our structural, biophysical, and biochemical data (Figure 3I). There is an extensive NTF/CTF interface with $2 \beta$ strands of the NTF interweaving with those of the CTF that is reinforced by multiple sidechain interactions. The loop containing the cleavage sites (cut 1, cut 2, cut 3) is solvent accessible and extends out of the NTF/CTF interface. The AlphaFold prediction further corroborates our experimental data and provides an atomistic model of the stable interaction between the NTF and CTF.

Overexpression of both cleaved and uncleaved CDCP1 induces downstream signaling. Overexpression of CDCP1 is associated with intracellular tyrosine phosphorylation and initiation of signaling pathways involving Src and PKC $\delta$ to promote protumorigenic processes, such as loss of adhesion and anoikis (30). To examine the function of the newly appreciated c-CDCP1 complex, we generated stable HEK293T cell lines expressing fl-CDCP1 or c-CDCP1 (Figure 4A). For fl-CDCP1, a lentiviral vector encoding the entire CDCP1 protein sequence was used. For c-CDCP1, we designed a vector in which a T2A self-cleaving sequence was placed between the CTF and the NTF. The T2A sequence was cleaved during translation to generate 2 polypeptides, enabling cell-surface expression of the c-CDCP1 complex from a single vector. We also generated variants where the 4 intracellular tyrosine residues were mutated to phenylalanine individually (Y707F, Y734F, Y743F, Y806F) or 
A

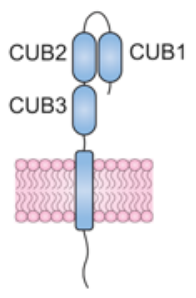

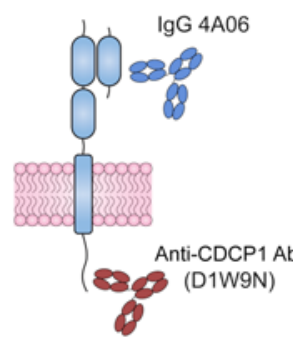
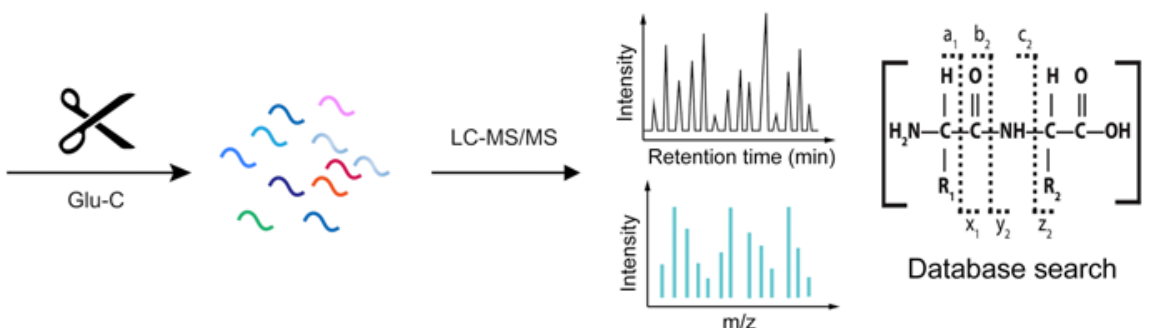

Database search

B

WB: CDCP1 (D1W9N)

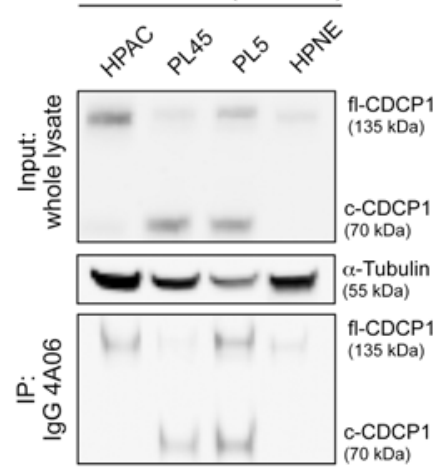

C PL5: MS-identified CDCP1 peptides

RAMSLTIEPRPVK 1 (K365)

RAMSLTIEPRPVKOSR Cut 3 (K369)

Reference sequence: SNKIYVVDLSNERAMSLTIEPRPVKOSRKFVPGCFVCLESRTCSSNLTLTSGSKHKISFLCDDLTRLWMNV

KFVPGCFVCL

FVPGCFVCL

PL45: MS-identified CDCP1 peptides

Cut 1 (K365)

RAMSLTIEPRPVK Cut 2 (R368)

RAMSLTIEPRPVKQSR| Cut 3 (K369)

Reference sequence: SNKIYVVDLSNERAMSLTIEPRPVKOSRKFVPGCFVCLESRTCSSNLTLTSGSKHKISFLCDDLTRLWMNV

HPAC: MS-identified CDCP1 peptides

KFVPGCFVCL

FVPGCFVCL

RAMSLTIEPRPVKQSRKFVPGCFVCL

Reference sequence: SNKIYVVDLSNERAMSLTIEPRPVKQSRKFVPGCFVCLESRTCSSNLTLTSGSKHKISFLCDDLTRLWMNV

Figure 2. Identification of endogenous cut sites of CDCP1 on the surface of PDAC cells. (A) Schematic of IP-MS strategy to identify the endogenous proteolysis sites of CDCP1 on PDAC cells. CDCP1 was immunoprecipitated with lgC 4A06 or D1W9N Ab and digested with Glu-C, which cleaves after aspartic acid. Liquid chromatography/tandem mass spectrometry (LC-MS/MS) was used to identify peptides corresponding to proteolytic products of CDCP1. (B) Top: Western blot of PDAC cell lines expressing differential amounts of uncleaved CDCP1 and c-CDCP1. D1W9N Ab was used to detect CTF of CDCP1. PL5 and PL45 express mostly c-CDCP1, while HPAC expresses mostly uncleaved CDCP1. HPNE, a nonmalignant pancreatic cell line, expresses low levels of CDCP1. Bottom: IP blot shows that IP of NTF with IgC 4 A06 can pull down the CTF of CDCP1. (C) CDCP1 peptides and proteolysis sites identified in PL5, PL45, and HPAC samples. Peptides identified by LC-MS for each cell line are aligned to the reference sequence (light blue). Glu- $C$ cleavage sites are in red underlined text. Three proteolysis sites of CDCP1, cut 1 (K365), cut 2 (R368), and cut 3 (K369) are identified in PL5 and PL45 cells, but are absent in HPAC cells.

together (4YF; ref. 30). Flow cytometry (Figure 4B) and Western blot (Figure 4C; see complete unedited blots in the supplemental material) confirmed the successful generation of these stable cell lines. We found that both fl-CDCP1 and c-CDCP1 and downstream signaling partners Src and PKC $\delta$ were phosphorylated in these cell lines (Figure 4C). Additionally, Y734 is critical for the phosphorylation of the other intracellular tyrosine residues of fl-CDCP1 and c-CDCP1 and of Src and PKC (30). Overexpression of either fl-CDCP1 or c-CDCP1 decreased cell adhesion and was dependent on intracellular tyrosine phosphorylation, specifically of Y734 (Figure 4D and Supplemental Figure 6), while there was no significant effect on cell growth (Supplemental Figure 7). Because the expression levels of fl-CDCP1 and c-CDCP1 are not the same, we are not able to directly compare phenotypic differences between fl-CDCP1 and c-CDCP1 in this context. Regardless, these results collectively show that the c-CDCP1 NTF/CTF complex appears functional and reflect the known biology of CDCP1.

IgG CLO3 specifically recognizes the cleaved form of CDCP1. To generate an $\mathrm{Ab}$ that can specifically recognize c-CDCP1, we employed a differential phage selection strategy using an in-house Fab-phage library (Figure 5A and ref. 31). Prior to each round of selection, the phage pool was cleared with fl-CDCP1 before positive selection with c-CDCP1. Purified antigens containing the 3 different cut sites were selected for individually or pooled. After 3 to 4 rounds of selection, there was enrichment for Fab-phage that bound c-CDCP1 over fl-CDCP1 (Supplemental Figure 8A). We identified a unique clone, CLO3, that bound all $3 \mathrm{c}-\mathrm{CDCP} 1$ antigens selectively over fl-CDCP1 with subnanomolar IgG affinity $\left(K_{D}\right.$ $=150-840 \mathrm{pM})($ Figure 5B, Supplemental Figure 8B, and Supplemental Table 2). Plasmin is reported to be one of the proteases that can cleave CDCP1 $(24,32)$, and we found that IgG CLO3 could also recognize plasmin-treated CDCP1 (Supplemental Figure 9, A-C).

We were interested in understanding how CLO3 differentiates between fl-CDCP1 and c-CDCP1. It is possible CLO3 could directly bind the cleavage "scars," but it was challenging to rationalize how the $\mathrm{Ab}$ could recognize all 3 different cut sites with similar affinity. Alternatively, CLO3 could bind an epitope that is unmasked upon proteolysis-induced conformational change. To investigate this, we tested the binding of CL03 to different CDCP1 constructs. We found that if the was immobilized via its C-termini, CL03 did not bind (Supplemental Figure 10, A and B). However, if the NTF was immobilized via its N-termini, CLO3 bound NTF with affinity similar to that of c-CDCP1 (Supplemental Figure 10, B and C). This suggests that the CLO3 epitope is located near the C-terminal portion of the NTF. Interestingly, we found that CLO3 can also recognize an uncleaved CDCP1 variant where a 16 aa linker is inserted between NTF and CTF at the R368/K369 site (Supplemental Figure 10D). This indicates that, akin to proteolysis, extending the loop between the NTF and CTF can also unmask the CLO3 epitope. 
A

Cotransfected strategy to generate cleaved CDCP1 ectodomain
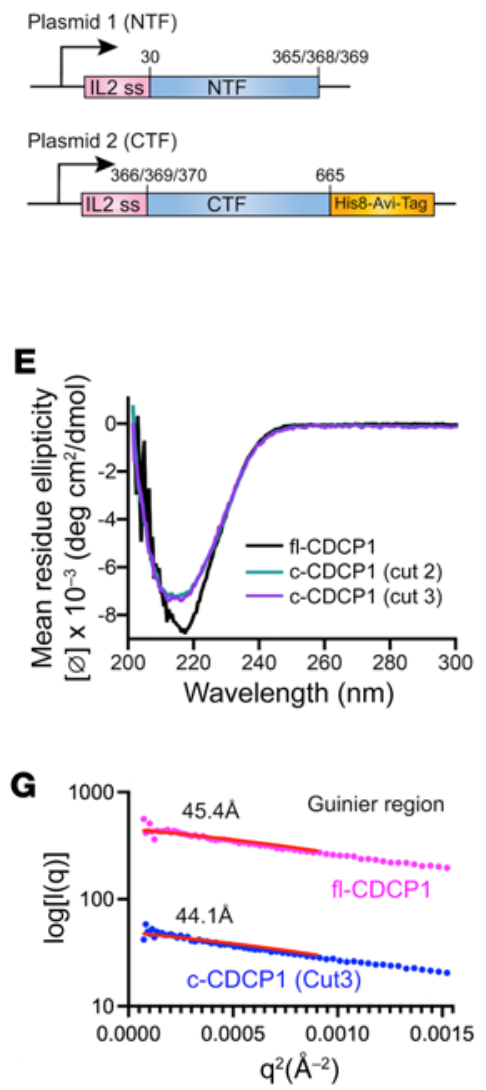
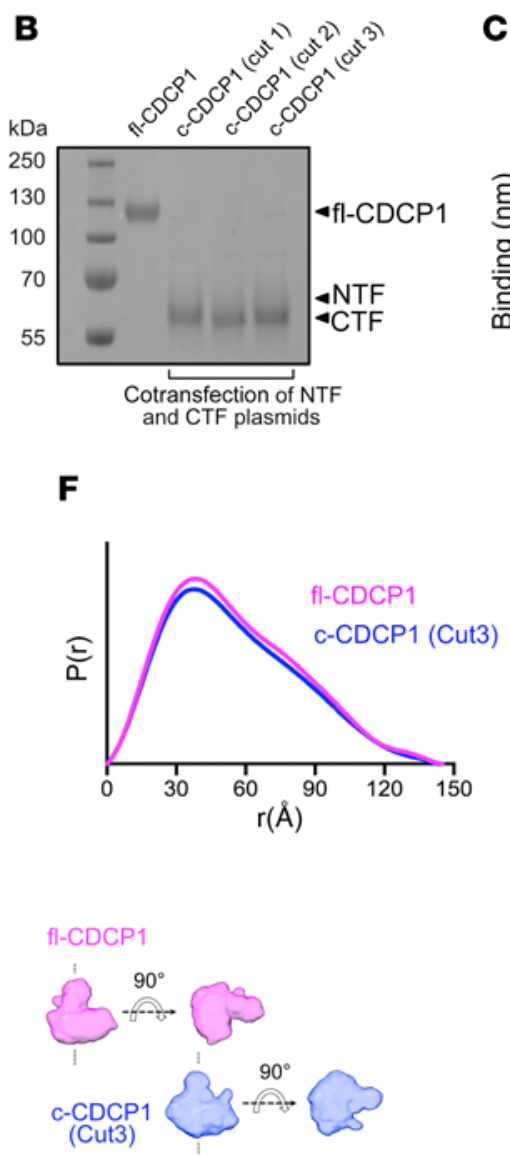
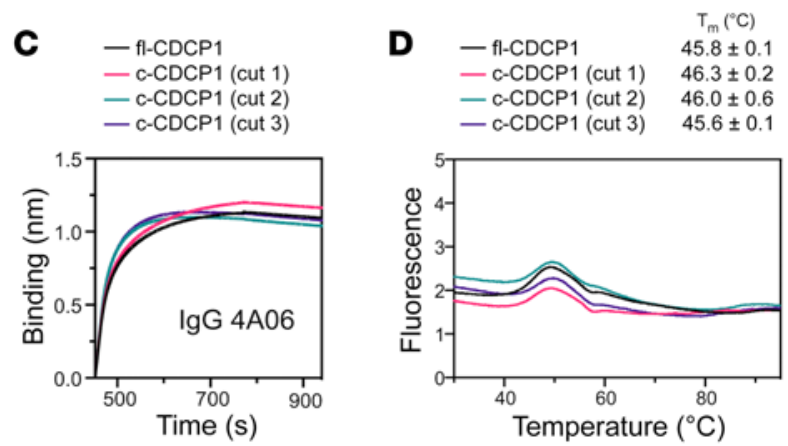

H
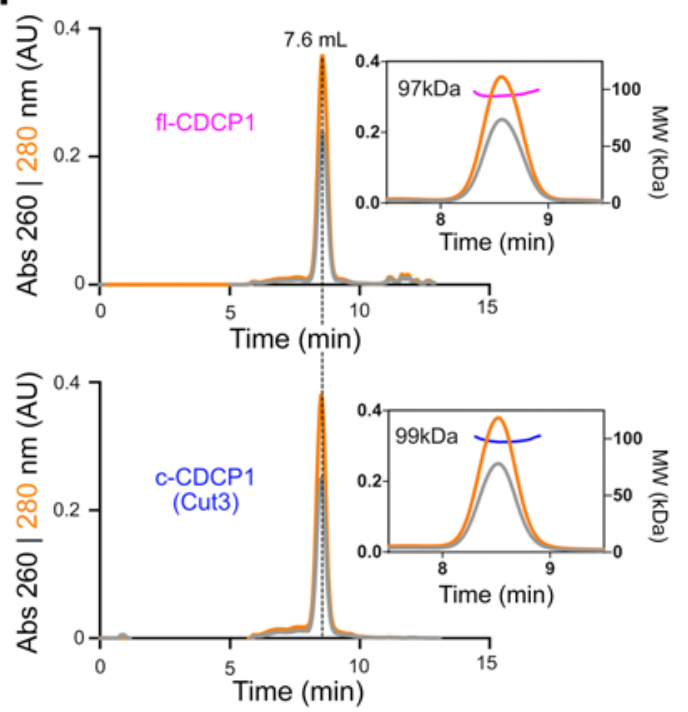
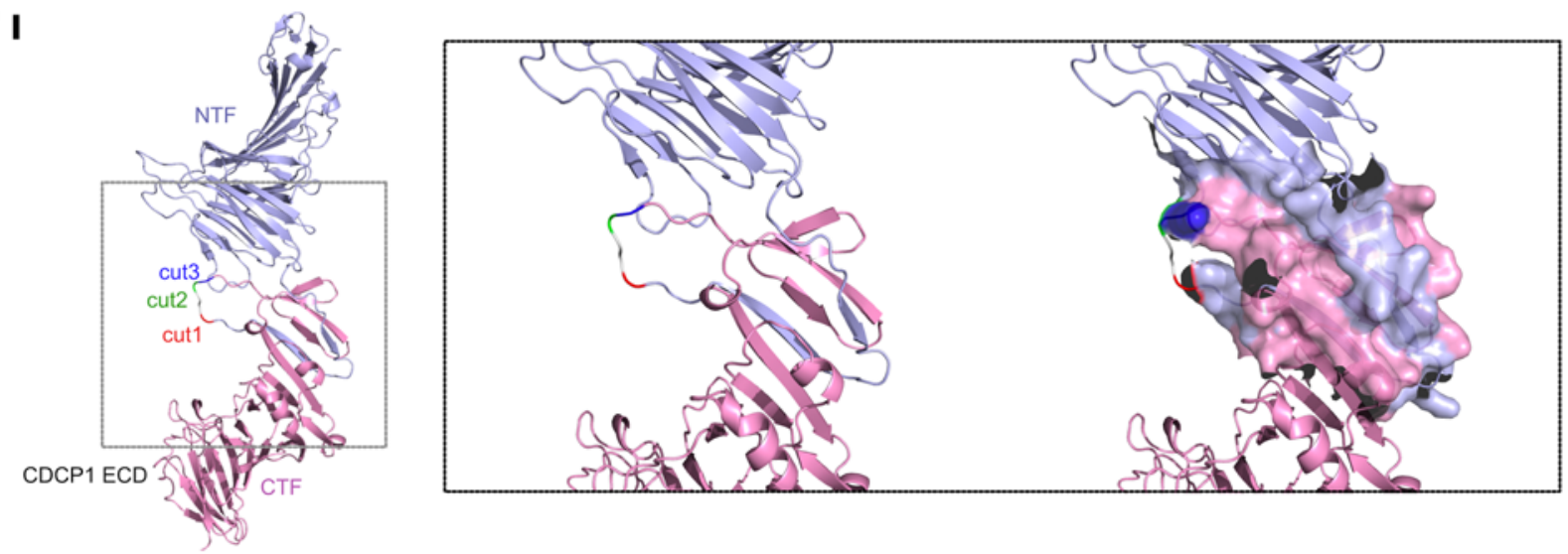

Figure 3. c-CDCP1 and uncleaved CDCP1 have similar conformations. (A) Schematic of the cotransfection strategy to generate c-CDCP1. The NTF and CTF are encoded on separate plasmids with an IL-2 signal sequence. (B) SDS-PAGE of fI-CDCP1 and c-CDCP1 (cut 1, cut 2, cut 3) ectodomain. NTF is heavily glycosylated and runs as a high-molecular weight smear. (C) BLI of IgC 4A06 to fl-CDCP1 or c-CDCP1 ectodomains shows that the NTF of CDCP1 is intact on c-CDCP1. (D) Differential scanning fluorimetry shows that fI-CDCP1 and c-CDCP1 have similar melting profiles and stabilities, suggesting that the NTF/CTF complex does not dissociate until unfolding of the full ectodomain. Melting temperature $\left(T_{m}\right)$ is reported as an average and SD of 2 replicates. (E) CD spectra of fl-CDCP1 and c-CDCP1. CDCP1 has a $\beta$ sheet signature with minima of approximately $217 \mathrm{~nm}$. The slight difference in spectral shape between fl-CDCP1 and c-CDCP1 indicates a subtle change in secondary structure. (F) SAXS-derived $P(r)$ function of fl-CDCP1 and c-CDCP1 ectodomains. (G) $R_{g}$ and SAXSderived ab initio envelopes of fl-CDCP1 and c-CDCP1 derived from SEC-SAXS show similar overall architecture. (H) SEC-MALS chromatograms of fl-CDCP1 and c-CDCP1 show similar elution profiles and molecular weights corresponding to monomeric ectodomain. (I) AlphaFold model of CDCP1 ectodomain. Residues involved in the NTF/CTF interface are shown as surface rendering in the inset. 
A Strategy to generate HEK293T expressing uncleaved and cleaved CDCP1

Cleaved CDCP1

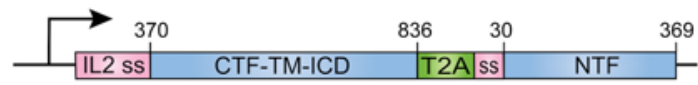

Uncleaved CDCP1

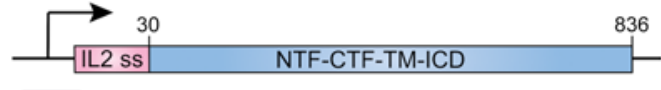

$\mathrm{T2A}$ = self-cleavage sequence

B

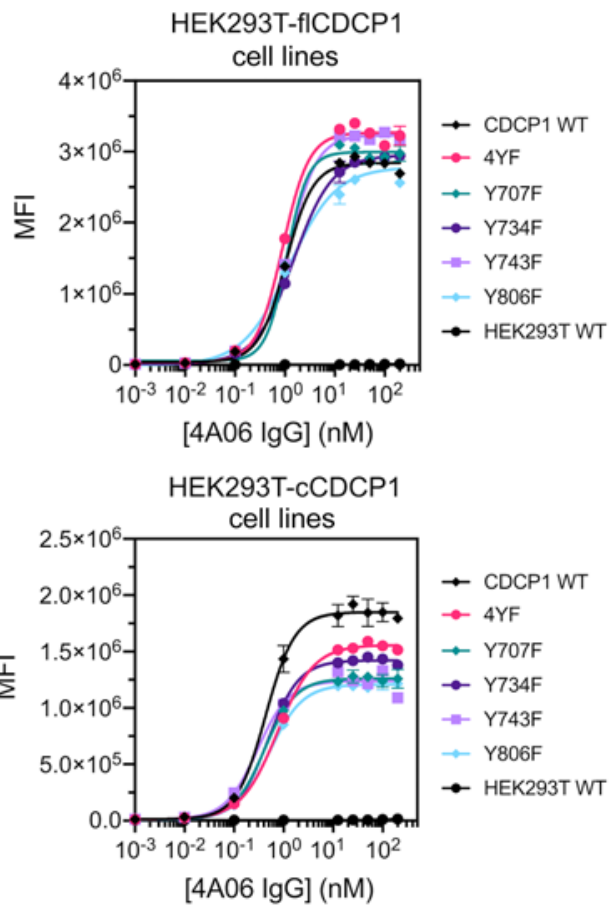

D

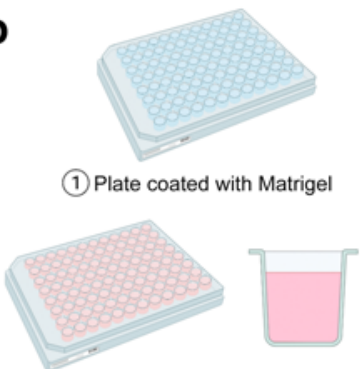

(2) Seed cells and incubate $2 \mathrm{~h}$

(3) Wash away non-adherent cells

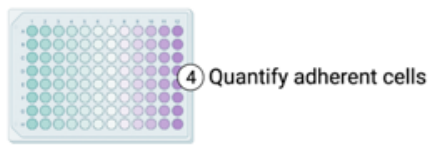

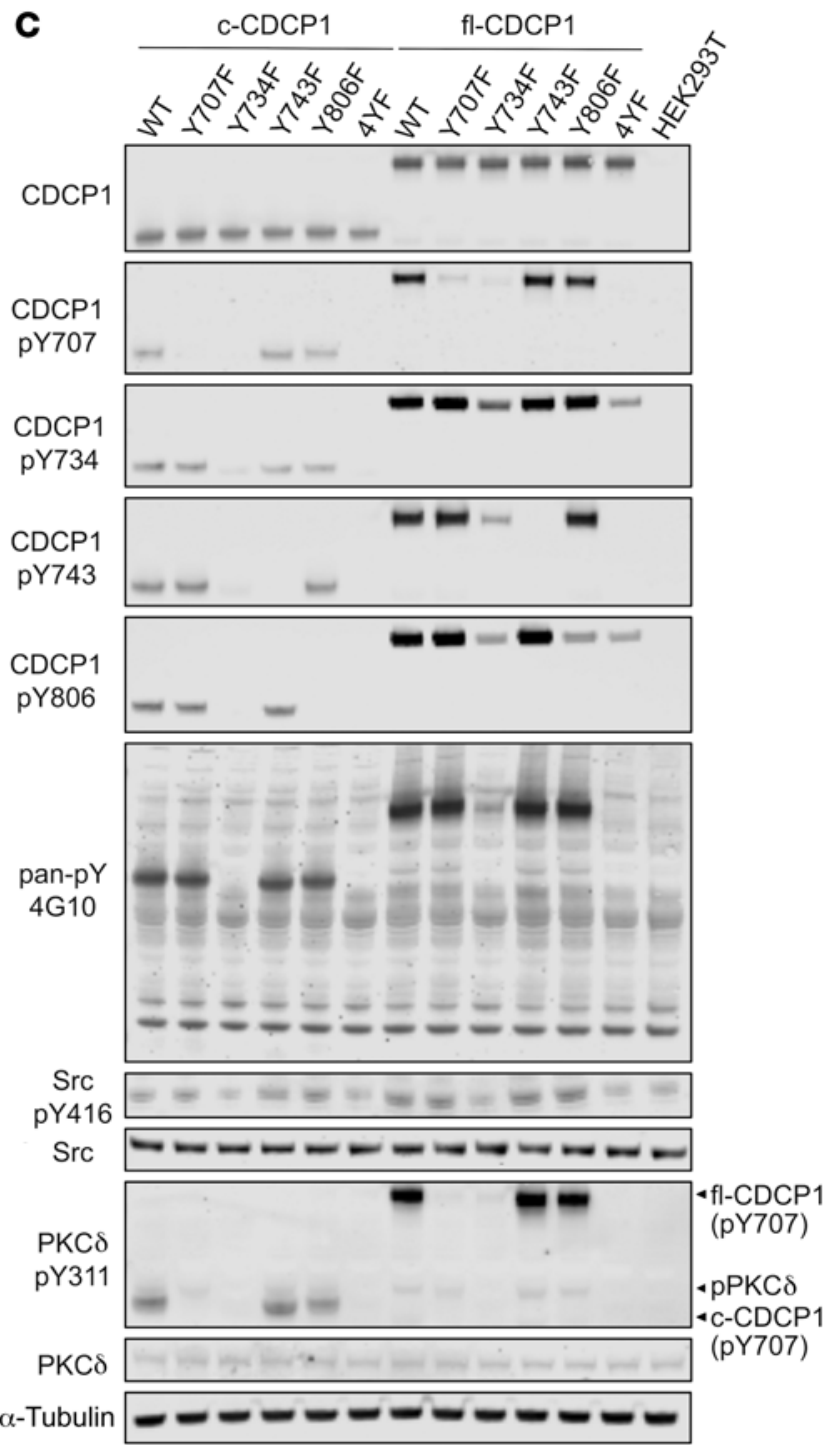

Cell adhesion assay
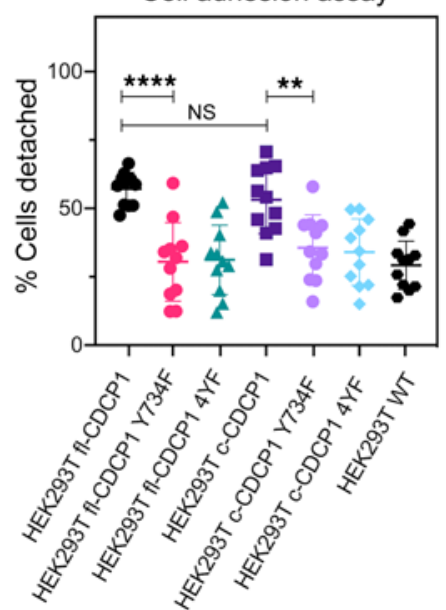
Figure 4. Both fl-CDCP1 and $\mathrm{C}-\mathrm{CDCP} 1$ induce signaling and promote loss of adhesion. (A) Schematic of strategy to generate HEK293T cell lines expressing fl-CDCP1 or C-CDCP1. For C-CDCP1, a lentiviral vector was designed where a T2A self-cleavage sequence flanks the CTF (residues 370-836) and NTF (residues 30-369). For fl-CDCP1, a lentiviral vector encoding the full CDCP1 sequence (residues 30-836) was designed. An IL-2 signal sequence precedes each fragment. (B) Flow cytometry of IgC 4A06 to HEK293T fl-CDCP1 and HEK293T c-CDCP1 cell lines. Data are represented as mean \pm SEM. $n=3$. (C) Western blot of CDCP1 and intracellular proteins associated with CDCP1 signaling. Both fl-CDCP1 and c-CDCP1 are phosphorylated and initiate downstream signaling mediated by Src and PKC $\delta$. Phosphorylation of Y734 on CDCP1 is important for phosphorylation of other tyrosine residues and Src and PKC $\delta$. Anti-phosphoY311-PKC $\delta$ appears to be crossreactive to CDCP1-pY734. (D) Cell-adhesion assay shows that overexpression of both fI-CDCP1 and c-CDCP1 decreases cell adhesion and is dependent on phosphorylation of intracellular tyrosine residues, specifically of Y734. Data represent individual values and mean \pm SEM. There was a significant difference in cell adhesion between the different cell lines $(F[6,70]=10.98, P<0.0001,1$-way ANOVA). Tukey's post hoc tests revealed that overexpression of either fl-CDCP1 or C-CDCP1 decreased cell adhesion $(P<0.0001$ for fl-CDCP1 vs. WT; $P=0.0001$ for c-CDCP1 vs. WT), but adhesion of HEK293T fl-CDCP1 and HEK293T c-CDCP1 was not significantly different (NS, $P=0.98$ ). The decreased cell adhesion was lost for both fl-CDCP1 and C-CDCP1 when all 4 intracellular tyrosine residues (4YF) or specifically Y734F was mutated $\left({ }^{* * *} P<0.0001\right.$ for fl-CDCP1 vs. fl-CDCP1 Y734F and 4YF, ${ }^{* *} P=0.011$ for c-CDCP1 vs. C-CDCP1 Y734F, $P=0.004$ for c-CDCP1 vs. C-CDCP1 4YF).

We obtained a 3D negative-stain electron microscopy (EM) reconstruction of c-CDCP1 ectodomain bound to CLO3 Fab at 25 Å resolution and bound to $4 \mathrm{A0} 6 \mathrm{Fab}$ at $23 \AA$ resolution (Figure $5 \mathrm{C}$ and Supplemental Figure 11). A nanobody that binds at the "elbow" of the light chain was used as a fiducial mark to determine the orientation and "handedness" of the Fab. (33) c-CDCP1 adopted an elongated structure with 3 distinct "lobes" of density. We reasoned from our binding data that the Fab-bound domain was the NTF and that the other lobes belonged to the CTF. CLO3 appeared to bind the NTF at a region proximal to the CTF, while 4 A06 bound at the apex of the NTF at a distinct, nonoverlapping epitope. The AlphaFold model of CDCP1 docked well into the negative-stain EM maps of both fl-CDCP1 and c-CDCP1 obtained in the presence of Fab 4A06 (Supplemental Figure 11F). Interestingly, the AlphaFold model fit less well to the c-CDCP1 density when Fab CLO3 was bound. It is possible that there are conformational rearrangements to c-CDCP1 that are induced by CLO3 binding. Taking these data together, we propose a model in which the epitope of CLO3 is located on the NTF proximal to the cut site, but is inaccessible in the uncleaved state. Proteolysis releases the C-termini of NTF to unmask this neoepitope and allow CLO3 to bind (Supplemental Figure 10E). This could be achieved by rearrangement of the secondary structure elements of c-CDCP1, even while adopting an overall conformation similar to that of the uncleaved form.

IgG CLO3 targets c-CDCP1-expressing PDAC cells. We then tested to determine whether our cleaved-specific Ab CLO3 can specifically recognize c-CDCP1 on cancer cells. IgG CLO3 stains c-CDCP1-expressing PL5 and PL45 cells with EC $_{50}$ values of 14.1 and $20.7 \mathrm{nM}$, respectively, with no detectable binding to HPAC, which expresses fl-CDCP1, or HPNE, which does not express CDCP1 (Figure 5, D and E). Treating HPAC cells with plasmin increased binding of IgG CLO3, suggesting that protease treat- ment can increase the amount of c-CDCP1 on the cell surface (Supplemental Figure 9, D and E). We then tested to determine whether an Ab-drug conjugate (ADC) strategy could be used to specifically deliver cytotoxic payloads to c-CDCP1-expressing PDAC cells. HPAC, PL5, PL45, and HPNE cells were treated with IgG CLO3 as the primary Ab along with a secondary Ab conjugated to cytotoxin monomethyl auristatin F (MMAF) (Figure 5F). We observed dose-dependent cell killing of only PL5 and PL45 cells, while HPAC and HPNE were spared. Next, we tested to determine whether CLO3, as a bispecific T cell engager (BiTE), could selectively recruit and activate immune cells in the presence of c-CDCP1-expressing target cells. Fab CLO3 was genetically fused to an anti-CD3 OKT3 scFv and incubated with a Jurkat NFAT-GFP reporter cell line in coculture with PDAC cells (Supplemental Figure 12). We observed a dose-dependent increase in Jurkat NFAT activation in coculture with PL5 and PL45 cells, while coculture with HPAC and HPNE resulted in only baseline activation. Finally, we investigated the in vivo tumor localization of ${ }^{89} \mathrm{Zr}$-radiolabeled IgG CLO3 in a PL5 mouse xenograft. PET imaging 48 hours after injection showed strong tumor localization of ${ }^{89} \mathrm{Zr}-\mathrm{IgG}$ CLO3 (Figure $5 G)$. Taken together, these studies demonstrate that IgG CLO3 can selectively target c-CDCP1-expressing pancreatic cancer cells in a variety of modalities both in vitro and in vivo.

IgG58, a cleaved-specific Ab to murine CDCP1, demonstrates antitumor activity with enhanced safety profile in a syngeneic mouse model. CLO3 is crossreactive to cynomolgus, but not to mouse c-CDCP1 (Supplemental Figure 13). To enable syngeneic studies, we utilized the same differential phage display selection strategy to identify surrogate Abs specific to mouse c-CDCP1 (Supplemental Figure 14, A-C). After characterization and affinity maturation, we arrived at a lead mouse cleaved-specific CDCP1 $\mathrm{Ab}, \mathrm{IgG58}$, which binds mouse c-CDCP1 with high affinity and specificity (Figure 6A and Supplemental Table 3). We also identified IgG12, which, akin to the human CDCP1-specific IgG 4A06, recognizes both mouse fl-CDCP1 and c-CDCP1 with similar affinities (Supplemental Figure 14D). We also generated a stable mouse c-CDCP1 cell line in the background of Fc1245, an aggressive KPC model (Figure 4A and ref. 34). Both IgG58 and IgG12 recognize Fc1245 c-CDCP1 with an $\mathrm{EC}_{50}$ of $6.9 \mathrm{nM}$ and $0.46 \mathrm{nM}$, respectively (Figure 6B and Supplemental Figure 14E). We further showed that IgG58 and IgG12, when reformatted to BiTE molecules, could activate Jurkat cells in the presence of Fc1245 c-CDCP1 cells (Supplemental Figure 14, F and G). Additionally, IgG58-MMAF and IgG12-MMAF ADC molecules could specifically deliver cytotoxic payloads to Fc1245 c-CDCP1 cells (Figure $6 \mathrm{C}$ and Supplemental Figure 14H).

We then tested the in vivo tumor localization of $\operatorname{IgG12}$ and IgG58. ${ }^{89} \mathrm{Zr}$-IgG12 or ${ }^{89} \mathrm{Zr}$-IgG58 was injected into mice harboring subcutaneous Fc1245 c-CDCP1 tumors that were examined 48 hours later by PET imaging (Figure 6, D and E). High tumor localization of ${ }^{89} \mathrm{Zr}$-IgG58 was observed, and this signal decreased with coadministration of $50 \times$ unlabeled cold $\operatorname{IgG58}$, indicating tumor-specific localization driven by specific target engagement. There was minimal ${ }^{89} \mathrm{Zr}-\mathrm{IgG} 58$ signal systemically. In contrast, we observed weaker tumor localization of ${ }^{89} \mathrm{Zr}-\mathrm{IgG} 12$ and more widespread off-tumor signal, indicating a higher presence of fl-CDCP1 in healthy tissues. 
A
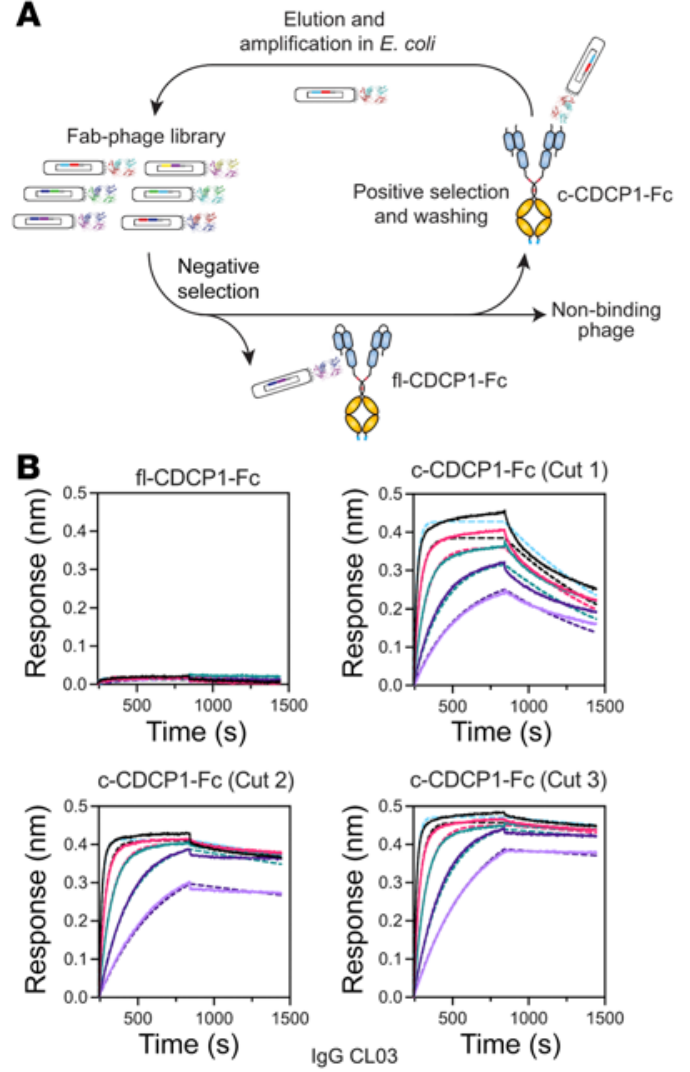

C

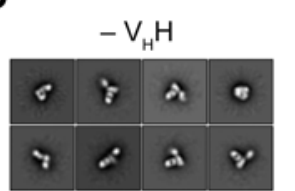

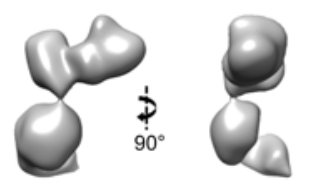
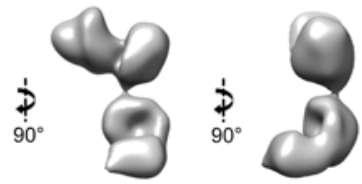

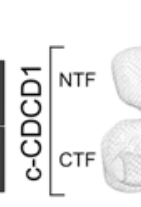

$\mathrm{V}_{\mathrm{H}} \mathrm{H}$
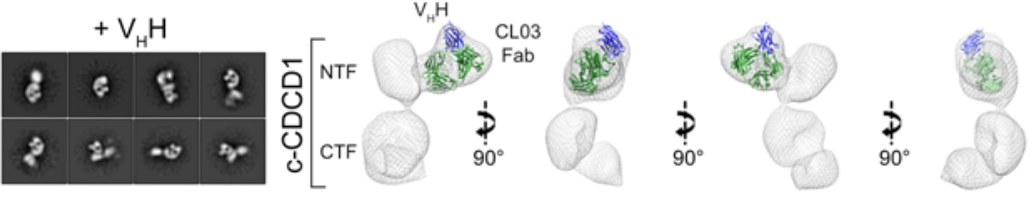

D
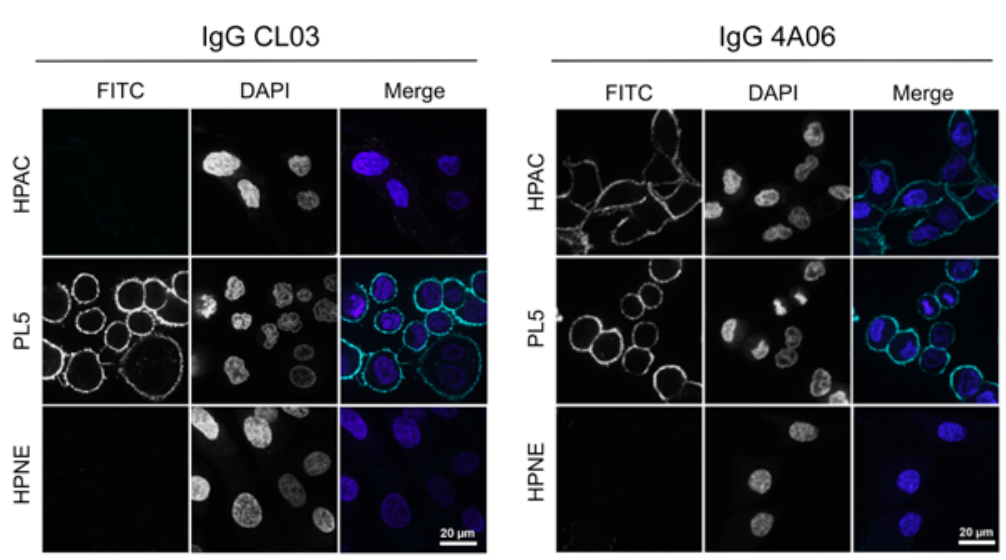

\section{E}

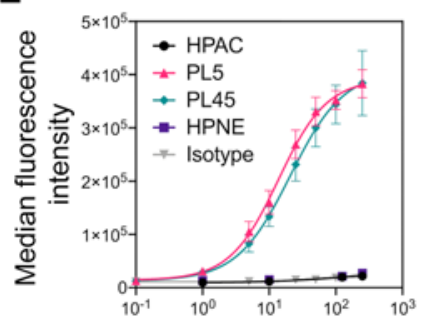

$[\mathrm{Ab}](\mathrm{nM})$

$\mathrm{EC}_{50}(\mathrm{PL5})=14.1 \mathrm{nM}(95 \% \mathrm{Cl}: 11.7-16.5)$ $\mathrm{EC}_{50}(\mathrm{PL} 45)=20.7 \mathrm{nM}(95 \% \mathrm{Cl}: 19.0-22.8)$

\section{$\mathbf{F}$}
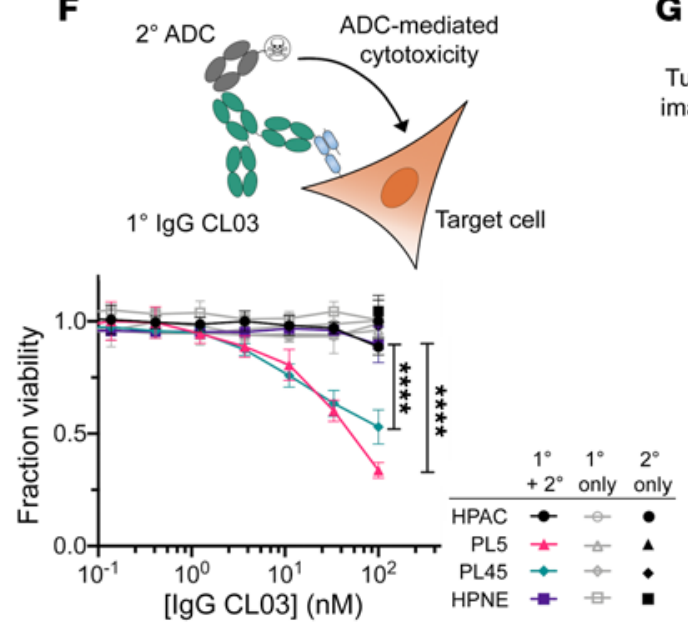
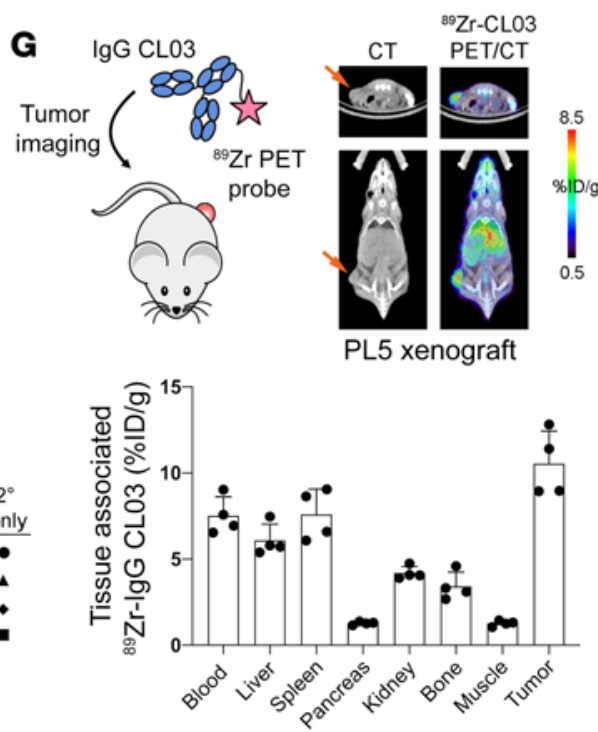

Figure 5. IgG CL03 specifically targets c-CDCP1-expressing pancreatic cancer cells. (A) Differential phage selection strategy to identify a c-CDCP1-specific Ab. Fab-phage was precleared with fl-CDCP1-Fc prior to positive selection with c-CDCP1-Fc. (B) BLI shows specific binding of IgG CL03 to c-CDCP1-Fc, but not to fl-CDCP1-Fc $\left(K_{D}=150-840\right.$ pM, Supplemental Table 1). (C) Negative-stain EM 3D reconstruction of c-CDCP1 with CL03 Fab. Left: 2D class averages of c-CDCP1 (cut 3) plus CL03 Fab in the absence and presence of anti-Fab $\mathrm{V}_{\mathrm{H}} \mathrm{H}$. Right: 3D EM maps of CDCP1 (cut 3) plus CL03 Fab plus $\mathrm{V}_{\mathrm{H}} \mathrm{H}$ with crystal structure of Fab (green) and $\mathrm{V}_{\mathrm{H}} \mathrm{H}$ (blue) modeled into the density. (D) Immunofluorescence of HPAC, PL5, and HPNE cells with Alexa Fluor 488 -labeled IgC CL03 (left panels) and IgG 4A06 (right panels). IgG CL03 specifically stains PL5 cells, while IgG 4A06 stains both HPAC and PL5 cells. No staining is observed for HPNE. Scale bars: $20 \mu \mathrm{m}$. (E) Flow cytometry shows that IgG CL03 binds to PL5 and PL45 cells, but not HPAC or HPNE cells. Data are represented as mean \pm SEM. $n=3$. (F) Top: schematic of ADC cell-killing assay. Bottom: dose-dependent ADC-mediated cell killing with lgG CL03 was only observed against PL5 and PL45 and only in the presence of both the primary and secondary Abs. Data are represented as mean \pm SEM. $n=3$. ${ }^{* * * *} P<0.0001,2$-way ANOVA. (G) In vivo PET imaging of ${ }^{89} \mathrm{Zr}$-labeled IgG CLO3 in PL5 mouse xenografts shows tumor localization. Data represent individual values and mean \pm SEM. $n=4$. 
A

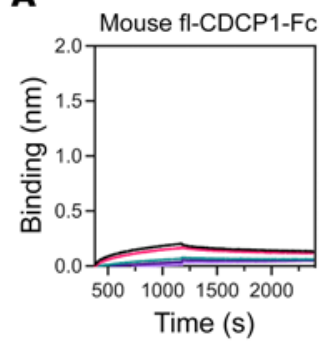

D
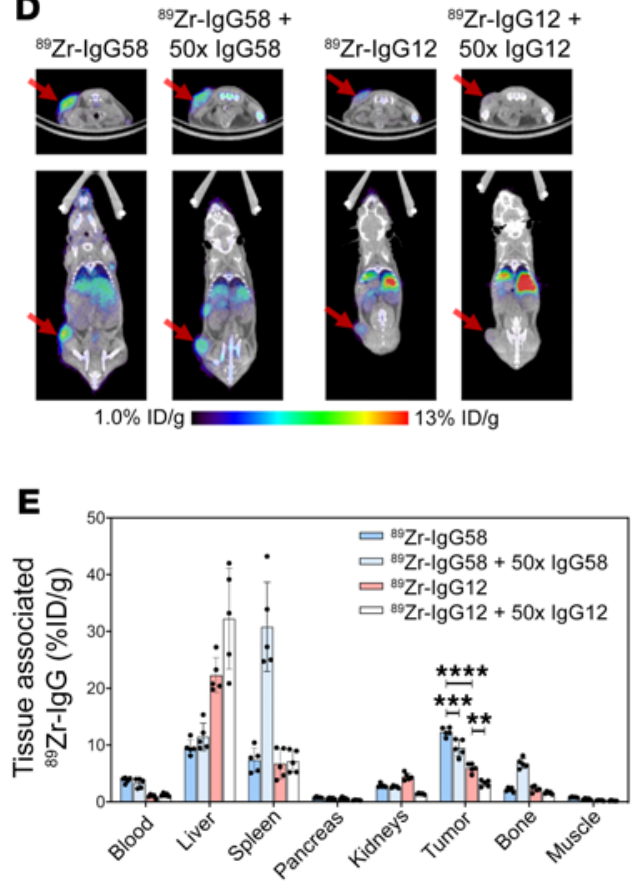
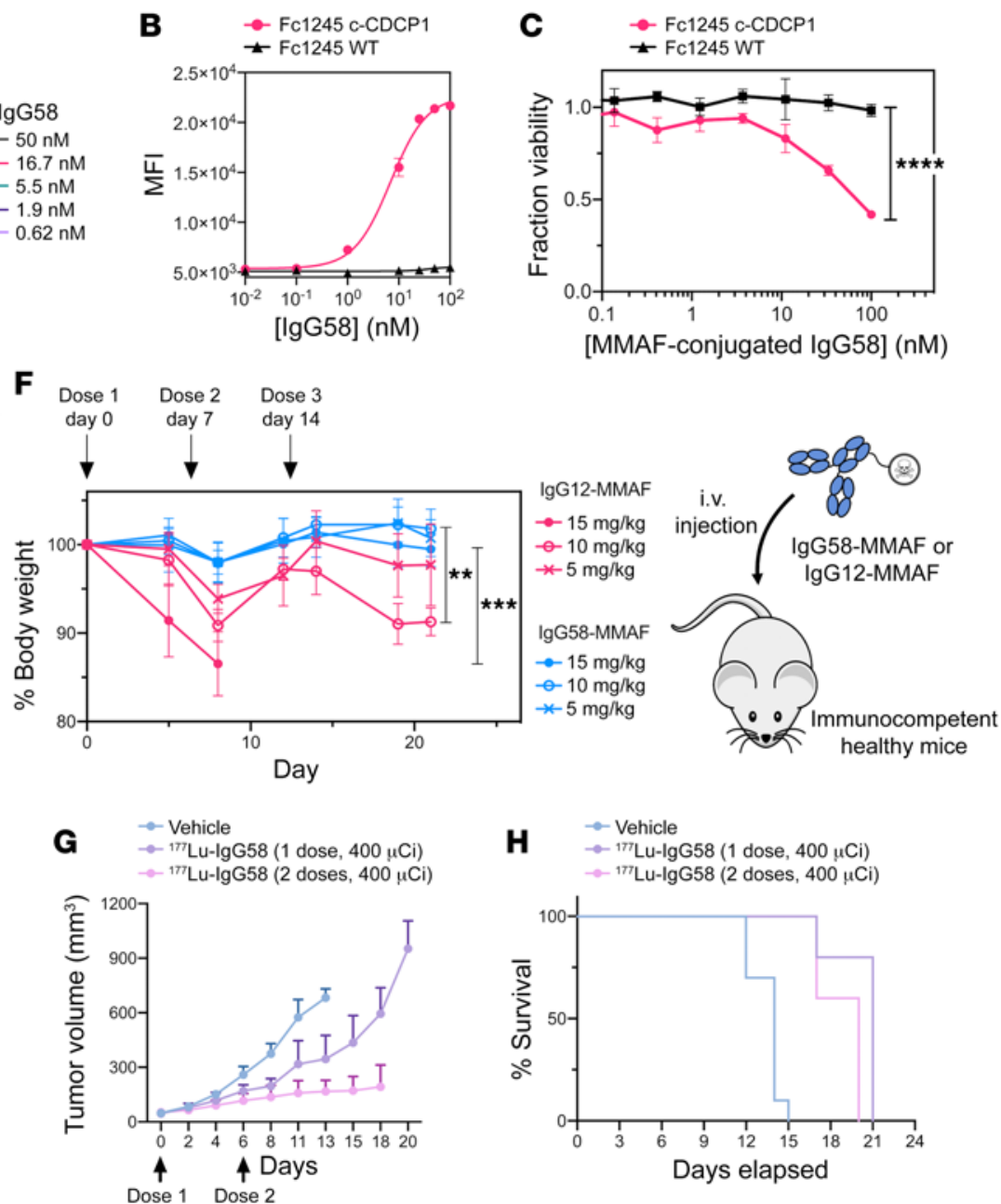

Figure 6. Efficacy of mouse c-CDCP1-specific Ab IgG58 in a syngeneic pancreatic tumor model. (A) BLI of IgG58 to mouse fl-CDCP1 and c-CDCP1. (B) Flow cytometry of IgG58. Data are represented as mean \pm SEM. $n=3$. (C) ADC-mediated cell killing of Fc1245 c-CDCP1 cells with IgG58-MMAF. Data are represented as mean \pm SEM. $n=3$. ${ }^{* * * *} P=0.0001$, 2-way ANOVA. (D) Representative in vivo PET images and (E) biodistribution of ${ }^{89} \mathrm{Zr}$-lgG58 and ${ }^{89} \mathrm{Zr}$-lgG12 in mice harboring s.c. Fc1245 c-CDCP1 tumors ( $n=5$ per group). Data represent individual values and mean \pm SEM. There was a significant difference in tumor signal across the treatment groups $\left(F[3,15]=95.11, P<0.0001\right.$, ANOVA). Tukey's post hoc tests revealed both ${ }^{89} \mathrm{Zr}$-lgG58 and ${ }^{89} \mathrm{Zr}$-lgG12 signal decreased with administration of $50 \times$ unlabeled IgG, indicating target-specific localization. ${ }^{* *} P=0.0005$ for IgG58; ${ }^{* *} P=0.0013$ for IgG12. There was significantly stronger tumor signal of ${ }^{89} \mathrm{Zr}$-lgG58 compared with ${ }^{89} \mathrm{Zr}$-lgG12, which shows weaker tumor localization and more widespread normal tissue distribution. ${ }^{* * * *} P<0.0001$. (F) ADC toxicity assay in non-tumor-bearing mice. Mice ( $n=5$ per arm) were dosed weekly with 5,10 , or $15 \mathrm{mg} / \mathrm{kg}$ of $\mathrm{IgG12}-\mathrm{MMAF}$ or IgG58-MMAF. There was a significant difference between the treatment arms ( $F[5,32]=3.11, P=0.0002$, ANOVA). IgG58-MMAF treatment was better tolerated, with significant differences between IgG12-MMAF and IgG58-MMAF treatments at the $15 \mathrm{mg} / \mathrm{kg}$ dose $\left({ }^{* * *} P=0.0068\right)$ and at the $10 \mathrm{mg} / \mathrm{kg}$ dose $\left({ }^{* *} P=0.0067\right)$ (Tukey's multiple comparisons test). Data are represented as mean \pm SEM. (G and $\left.\mathbf{H}\right)$ Theranostic study of ${ }^{177}$ Lu-lgG58 $(n=5$ mice per treatment arm, $n=8$ for vehicle arm). Treatment with $400 \mu \mathrm{Ci}$ per dose of ${ }^{177} \mathrm{Lu}$-IgG58 resulted in decreased tumor growth and increased survival compared with vehicle. Data are represented as mean \pm SEM. There was a statistically significant difference in tumor volume between vehicle group vs. ${ }^{177} \mathrm{Lu}$-IgG58 1-dose group $(P=0.0008)$ and vehicle group vs. ${ }^{177} \mathrm{Lu}$-IgG58 2-dose group $(P<0.0001)$, unpaired 2 -tailed $t$ test.

We proceeded to examine the safety profile of targeting cleaved versus uncleaved CDCP1. Non-tumor-bearing mice were dosed weekly with 5,10 , or $15 \mathrm{mg} / \mathrm{kg}$ of IgG12-MMAF or IgG58MMAF, and their body weight was monitored for 21 days. None of the mice that received IgG58-MMAF at the 3 different doses exhibited significant changes in body weight (Figure 6F). In contrast, mice treated with IgG12-MMAF experienced significant body weight loss following each dose, indicative of treatmentinduced toxicity. All mice receiving the $15 \mathrm{mg} / \mathrm{kg}$ dose of IgG12MMAF had to be euthanized due to body weight loss by day 8 , and 2 of the 5 mice receiving the $10 \mathrm{mg} / \mathrm{kg}$ dose of IgG12-MMAF were euthanized on day 19. These toxicity results suggest that a $\mathrm{c}-\mathrm{CDCP} 1 \mathrm{Ab}$ would have a superior safety profile compared with a pan-CDCP1 targeting approach.

Finally, we investigated the antitumor activity of IgG58 as a radioligand therapeutic. Treatment of mice harboring subcutaneous Fc1245 c-CDCP1 tumors with 1 or $2400 \mu \mathrm{Ci}$ doses of ${ }^{177} \mathrm{Lu}$-IgG58 resulted in significantly reduced tumor volume compared with vehicle control, with the 2-dose regimen approaching tumor stasis (Figure 6G and Supplemental Figure 15). Median survival for the treatment arms were 21 and 20 days for the 1and 2-dose regimen, respectively, compared with 14 days for the 
vehicle group. The significant survival advantage imparted by ${ }^{177} \mathrm{Lu}-\mathrm{IgG} 58 \mathrm{c}-\mathrm{CDCP} 1$ theranostic therapy (Figure 6H) supports our conclusion that Abs specific to proteolytic neoepitopes could expand the targetable disease space for cancer treatment. Compared with subcutaneous models, orthotopic mouse models are known to better simulate clinical prostate cancer, particularly with respect to the gene expression profiles and tumor microenvironment. In spite of little difference between these 2 models for extremely aggressive Fc1245 tumors, an orthotopic animal model is obviously the next step.

\section{Discussion}

CDCP1 was first identified as a highly upregulated gene in colorectal and lung cancer (13) and has emerged as a driver of tumorigenesis and metastasis across a wide range of indications $(12,17$, $20,35)$. CDCP1 has been linked to a variety of oncogenic signaling networks, including Ras, EGFR, PDGFR, HER2, and $\operatorname{HIF}(15,36$, 37). Therapeutic interest in CDCP1 is reflected in numerous studies that report on small molecules (38) and Abs (16, 19, 32, 39-41) against CDCP1 and its pathways. However, despite these efforts, an anti-CDCP1 therapeutic has yet to enter the clinic. Although CDCP1 is highly expressed on cancer cells at close to approximately 2 million copies per cell $(15,16)$, it is also present on normal epithelial tissue (42). There is evidence that CDCP1 is not cleaved during normal physiological processes, but its cleavage is induced during tumorigenesis (43). This and other reports (25-27) suggest that c-CDCP1 would be rare on the surface of normal cells, and our work demonstrates that selectively targeting c-CDCP1 could be a safer, more therapeutically attractive approach. Continued work to characterize the prevalence and role of c-CDCP1, particularly in clinically relevant samples, will help determine which patient populations would be best suited to a c-CDCP1-targeting strategy.

Interestingly there is remarkably little conformational change between c-CDCP1 and fl-CDCP1. This presents a model for CDCP1 proteolysis and has been recently corroborated by Kryza et al. (44), who also observed that the NTF of CDCP1 does not dissociate upon proteolysis. By determining the exact sites of proteolysis and utilizing multiple biophysical and biochemical methods on both recombinant and cell lines, we further bolster the evidence that c-CDCP1 forms a complex and has a conformation similar to that of fl-CDCP1. Our structural, biophysical, and biochemical data are consistent with the structure predicted by AlphaFold and collectively provide what we believe is the most detailed structural examination of CDCP1 to date (29). The interweaving $\beta$ sheets of the NTF and CTF form a highly stable $\beta$ sheet bundle and an extensive interaction interface with the proteolysis site adjacent to this interface. We believe this model supports our findings that (a) upon proteolysis, the NTF stays tightly associated to the CTF, (b) there is little conformational change upon proteolysis, and (c) the CTF does not express in the absence of the NTF, due to the absence of the interweaved $\beta$ sheet interactions to form a properly folded CTF. Furthermore, although CDCP1 has previously been predicted to have 3 CUB domains, the AlphaFold model shows that the NTF is composed of a single domain that does not adopt a CUB domain fold, while the CTF contains 2 CUB-like domains.

Extracellular neoepitopes generated by alternate splice forms, MHC-peptide complexes, posttranslational modifications (PTMs), and glycosylation are emerging as classes of therapeutic targets for cancer and other disease. However, characterizing these neoepitopes and developing therapeutic molecules that can selectively recognize them are challenging. Complications include, but are not limited to, low abundance of MHC-peptide complexes, rarity of alternative splice variants with targetable epitopes, glycoform heterogeneity, and identifying and validating truly diseasespecific proteoforms. Proteolysis provides unique advantages in that it is irreversible, is highly prevalent in the tumor microenvironment, and can alter the conformation and structure of proteins and protein complexes in both subtle and marked ways. We show that proteolysis-generated neoepitopes on the cancer cell surface can provide an orthogonal approach to expanding the therapeutic index. The cleaved-specific CDCP1 Abs described here demonstrate that specifically targeting c-CDCP1 is effective and has a more favorable safety profile compared with targeting pan-CDCP1. Our work is a demonstration of an Ab specific to a proteolytically processed form of a cancer-associated cell-surface protein. Given the important and widespread role of proteases in disease biology, proteolysis-induced neoepitopes are likely widespread and could be targeted with Abs using a similar strategy. Recent unbiased proteomics methods that allow for detailed characterization of proteolysis on the cell surface will greatly increase the identification of disease-associated proteolytic neoepitopes (6).

Several serine proteases, such as plasmin $(19,28)$, matriptase (45), and UPA (28), have been shown to cleave CDCP1, are upregulated in solid tumors, such as pancreatic cancer (46), and are found in high levels in human clinical samples (47). Understanding the contributions of these proteases in the tumor microenvironment and any differences in activity and expression levels in patient subpopulations will inform our future strategy for proteolysis-targeted therapeutics. Solid tumors pose multiple therapeutic hurdles from a paucity of tumor-specific antigens, immunosuppressive microenvironment, hypoxia, and a complex stromal architecture (48). We anticipate that investigating these enhanced tumor-selective proteolysis markers, in combination with other traditional and emerging immunotherapy approaches (49), is likely to demonstrate the most efficacy and therapeutic benefit for patients.

\section{Methods}

Cloning, protein expression, and purification. CDCP1, IgGs, and BiTEs were cloned into pFUSE. Fabs were subcloned into pBL347. pCDH-EF1CymR-T2A-Neo was used for stable cell line cloning. Sequences were confirmed by Sanger sequencing. We used a previously described method for expression and purification of Fabs (31). CDCP1, IgGs, and BiTEs were generated by transfection of BirA-Expi293 cells using the ExpiFectamine 293 Transfection Kit (Life Technologies), purified by protein A or Ni-NTA affinity chromatography, and assessed by SDS-PAGE.

Lentiviral cell line construction. Stable cell lines were generated by lentiviral transduction. HEK293T Lenti-X cells were transfected with second-generation lentiviral packaging plasmids at approximately $80 \%$ confluence. FuGene HD (Promega) was used for transfection. After 72 hours, supernatant was harvested and filtered. Cleared supernatant was added to target cells with polybrene and centrifuged at $1000 \mathrm{~g}$ at $33^{\circ} \mathrm{C}$ for 2 hours. Cells were incubated with viral supernatant overnight before the media was changed to fresh com- 
plete DMEM. Cells were expanded for 48 hours before being grown in drug-selection media. After 72 hours, cells were analyzed by flow cytometry for expression.

Mammalian cell culture. HPAC, PL5, PL45, and HPNE cells were a gift from the laboratory of E. Scott Seeley (Stanford University, Stanford, California, USA) and were maintained in IMDM plus 10\% FBS plus $1 \times$ penicillin/streptomycin. HEK293T and Fc1245 cell lines were cultured in DMEM plus 10\% FBS plus 1× penicillin/streptomycin. Jurkat NFAT-GFP cell lines were cultured in RPMI plus 10\% FBS plus 2 $\mathrm{mg} / \mathrm{mL}$ G418 plus 1× penicillin/streptomycin.

$I P$. Cells were washed with PBS and lysed with ice-cold NP-40 with protease inhibitor cocktail (Roche) and PhosSTOP (Roche). The lysate was incubated at $4^{\circ} \mathrm{C}$ for 30 minutes and centrifuged at 14,000 $g$ for 30 minutes at $4^{\circ} \mathrm{C}$. For IP, CDCP1 Abs (D1W9N, Cell Signaling) were added to cell lysate precleared by protein A magnetic beads (EMD Millipore) and incubated overnight at $4^{\circ} \mathrm{C}$. Abs were captured with protein A magnetic beads. For Western blot, $4 \times$ SDS loading buffer was added to beads and heated at $95^{\circ} \mathrm{C}$ for 5 minutes to elute protein. For IP-MS, protein was eluted with $0.1 \mathrm{M}$ acetic acid and neutralized with $\mathrm{pH} 11$ Tris.

MS. Samples were reduced with TCEP and alkylated with iodoacetamide. Proteins were digested with $20 \mu$ g sequencing-grade Glu-C (Promega) in $1 \mathrm{M}$ urea at $37^{\circ} \mathrm{C}$ overnight. The samples were desalted using a Sola column (Thermo Fisher), dried, and dissolved in $0.1 \%$ formic acid plus $2 \%$ acetonitrile; $1 \mu \mathrm{g}$ of peptide was injected into a prepacked $0.075 \mathrm{~mm} \times 150 \mathrm{~mm}$ Acclaim Pepmap C18 LC column (2 $\mu \mathrm{m}$ pore size, Thermo Fisher) attached to a $\mathrm{Q}$ Exactive Plus (Thermo Fisher) mass spectrometer. Peptides were separated using a linear gradient of 3\%-35\% solvent B (solvent A: $0.1 \%$ formic acid; solvent B: $80 \%$ acetonitrile, $0.1 \%$ formic acid) over 170 minutes at $300 \mu \mathrm{L} /$ min. MS1 and MS2 scans were collected in data-dependent acquisition mode using a top-20 method with a dynamic exclusion of 35 seconds and a charge exclusion restricted to charges of 2, 3, or 4. Peptide search and MS1 peak area quantification were performed using ProteinProspector (version 5.13.2).

Western blot. Immunoblotting was performed using the following antibodies: CDCP1 (D1W9N) (catalog 13794S), phospho-CDCP1 (Tyr707) (catalog 13111S), phospho-CDCP1 (Tyr806) (catalog 13024S), phospho-CDCP1 (Try734) (catalog 9050S), phosphoCDCP1 (Try743) (D2G2J) (catalog 14965S), Src (36D10) (catalog 2109S), phospho-Src family (Tyr416) (catalog 2101S), PKC $\delta$ (cata$\log$ 2058S), phospho-PKC $\delta$ (Tyr311) (catalog 2055S), and $\alpha$-tubulin (DM1A) (catalog 3873S), imaged with LiCOR IRDye 680RD goat antimouse (catalog 925-68070), and IRDye 800CW goat anti-rabbit (catalog 926-32211) (all from Cell Signaling Technology).

Flow cytometry. Cells were lifted with Versene. Primary Abs were added for 30 minutes at $4^{\circ} \mathrm{C}$ and detected with the addition of Alexa Fluor 488 or Alexa Fluor 647-conjugated goat anti-human IgG, $\mathrm{F}(\mathrm{ab}$ ')2 fragment specific (Jackson ImmunoResearch). Cells were analyzed using a CytoFLEX (Beckman Coulter) flow cytometer. All flow cytometry data analysis was performed using FlowJo software, version 10.8.1, and Prism software, version 9.3.1 (GraphPad).

$B L I$. BLI was performed using an Octet RED384 instrument (FortéBio). Biotinylated proteins were immobilized on a streptavidin (SA) biosensor, and His-tagged proteins were immobilized on a Ni-NTA biosensor. Affinities were calculated from a global fit (1:1) of the data using Octet RED384 data analysis software, version 12.0.
SEC. SEC was performed using an Agilent HPLC 1260 Infinity II LC System using an AdvanceBio SEC column (300, $2.7 \mu \mathrm{m})$ in 0.15 $\mathrm{M}$ sodium phosphate. Each analyte was injected at 1 to $10 \mu \mathrm{M}$. Absorbance at $280 \mathrm{~nm}$ was monitored.

CD spectroscopy. CD spectra were measured using an Aviv 410 CD spectrophotometer. The CD signal from $200 \mathrm{~nm}$ to $300 \mathrm{~nm}$ was collected in a $0.1 \mathrm{~cm}$ path length cuvette at $25^{\circ} \mathrm{C}$.

SEC-SAXS and SEC-MALS. SEC-SAXS data were collected at the SIBLYS beamline 12.3.1 of the Advanced Light Source at the Lawrence Berkeley National Laboratory (Berkeley, California, USA). Data were collected using a Dectris PILATUS3 $2 \mathrm{M}$ detector at $20^{\circ} \mathrm{C}$ and processed as previously described (50). The SEC-SAXS flow cell was directly coupled with an Agilent 1260 Infinity HPLC System using a Shodex KW-803 column. The column was equilibrated with PBS pH 7.4 at $0.45 \mathrm{~mL} / \mathrm{min} ; 50 \mu \mathrm{L}$ of protein was injected at approximately $5 \mathrm{mg} / \mathrm{mL}$, and 3-second x-ray exposures were collected continuously. Radius of gyration $\left(R_{g}\right)$ was determined based on the Guinier approximation. Interference-free SAXS curves with least $R_{g}$ variation were averaged and merged in ScÅtter to produce the highest signal-to-noise SAXS curves. Pair distribution $P(r)$ function was computed using program GNOM (51). $P(r)$ functions were normalized based on molecular weight as determined based on their calculated constant volume $\left(V_{c}\right)$. MALS were collected using an 18-angle DAWN HELEOS II light scattering detector connected in tandem to an Optilab refractive index concentration detector (Wyatt Technology). MALS and differential refractive index data were analyzed using Wyatt Astra 7 software.

Differential scanning fluorimetry. $2 \mu \mathrm{M}$ protein in PBS was mixed with $4 \times$ Sypro Orange dye in a Bio-Rad 384-well PCR white plate and covered with qPCR Sealing Tape. The assay was performed over $25^{\circ} \mathrm{C}$ to $95^{\circ} \mathrm{C}$ with a temperature ramp rate of $0.5^{\circ} \mathrm{C} / 30 \mathrm{~s}$ on a Roche LC480 Light Cycler.

Negative-stain EM. Complexes of c-CDCP1 with CLO3 Fab and $4 \mathrm{AO} 6 \mathrm{Fab} \pm \mathrm{V}_{\mathrm{H}} \mathrm{H}$ were obtained by SEC on a Superdex 200 increase 10/300 GL column in $10 \mathrm{mM}$ HEPES, $100 \mathrm{mM} \mathrm{NaCl}, \mathrm{pH} 7.5 ; 5 \mu \mathrm{L}$ of protein sample at $0.006-0.008 \mathrm{mg} / \mathrm{mL}$ was applied on a glow-discharged formvar/carbon-coated TEM grid and stained using a sequential 4-droplet method with $1 \%$ uranyl formate. Grids were screened on a FEI Tecnai G2 F30 300 kV Super Twin TEM Electron Microscope (FEI Company) at the Advanced Electron Microscopy Facility at the University of Chicago (Chicago, Illinois, USA). Micrographs were acquired at magnification $49 \mathrm{kX}$ with a pixel size of $0.23 \mathrm{~nm}$ on the level of specimen using a $4 \mathrm{~K} \times 4 \mathrm{~K}$ CCD camera. Particles were selected automatically using RELION (52). Extracted particles were 2D class averaged, sorted into initial classes, 3D classified, and refined in RELION. Final maps were analyzed in Chimera (53).

Cell proliferation assay. Cell proliferation assays were performed using a modified MTT assay to measure cell viability. 5000 Cells/well were plated in a 96 -well plate on day 0 and incubated at $37^{\circ} \mathrm{C}$ under $5 \% \mathrm{CO}_{2} ; 10 \mu \mathrm{L}$ of $5 \mathrm{mg} / \mathrm{mL}$ of Thiazolyl Blue Tetrazolium Bromide (Sigma Aldrich) was added to each well and incubated at $37^{\circ} \mathrm{C}$ for 2 hours, and $100 \mu \mathrm{L}$ of $10 \%$ SDS plus $0.01 \mathrm{M} \mathrm{HCl}$ was added to lyse the cells to dissolve the MTT product. After 4 hours, absorbance at 595 $\mathrm{nm}$ was measured using an Infinite M200 PRO-Plate Reader (Tecan).

Cell adhesion assay. On day 1, a 96-well tissue culture plate was coated with MaxGel ECM (MilliporeSigma) 1:10 diluted in serum-free DMEM. The culture medium for HEK293T cells was also changed to serum-free medium. The next day, media was removed and culture 
plates were blocked with $100 \mu \mathrm{L}$ serum-free DMEM with $0.1 \%$ BSA for 2 hours and washed with PBS; 100,000 cells in $100 \mu \mathrm{L}$ of serum-free $(0.1 \%$ BSA $)$ medium were added to each well and incubated at $37^{\circ} \mathrm{C}$ for 2 hours. The nonadherent cells were removed by washing with media 3 times, and the remaining cells were quantified by MTT assay.

Phage selection and ELISA. All phage selections were conducted according to previously established protocols (31). Briefly, selections were performed using biotinylated c-CDCP1-Fc captured on SA-coated magnetic beads (Promega). Prior to each selection, the phage pool was incubated with biotinylated fl-CDCP1-Fc captured on SA beads. Four rounds of selection were performed with decreasing amounts of antigen. Bound Fab-phage was eluted by the addition of TEV protease. Individual clones from the third and fourth round of selection were analyzed by phage ELISA. Phage ELISA was performed according to standard protocols and detected using HRP-conjugated anti-M13 phage Abs (GE Lifesciences, 27-9421-01) using a TMB substrate.

Immunofluorescence. HPAC, PL5, and HPNE cells were plated on glass-bottom imaging plates (MatTek) and incubated for 24 hours at $37^{\circ} \mathrm{C}$. Cells were treated with $\operatorname{IgG}(1 \mu \mathrm{g} / \mathrm{mL})$ for 30 minutes and washed with media to remove unbound IgG. Bound IgG was detected by the addition of Alexa Fluor 488-conjugated AffiniPure $F\left(\mathrm{ab}^{\prime}\right) 2$ fragment goat anti-human IgG, $\mathrm{F}\left(\mathrm{ab}^{\prime}\right) 2$ fragment specific (Jackson ImmunoResearch, 143225), in Invitrogen Molecular Probes Live Cell Imaging Solution (Thermo Fisher) containing Hoescht blue $(2 \mu \mathrm{g} /$ $\mathrm{mL}$ ). Cells were imaged on a Nikon Ti Microscope Yokogawa CSU-22 with spinning disk confocal.

In vitro $A D C$ assays. Secondary $A D C$ assays used a Fab anti-human IgG Fc-MMAF Ab with cleavable linker (Moradec). For ADC assays using direct conjugation, the Ab was labeled with DBCO-PEG4-ValCitMMAF (Levena Biosciences) at residue T74M of the light chain using oxazirdine chemistry following a previously described protocol (54). 5000 Cells/well were seeded on a 96-well polylysine-coated white plate. The next day, media was removed and primary IgG and secondary ADC at a 1:4 ratio or MMAF-labeled IgG was added. Cells were incubated for 72 hours, and viability was measured using CellTiterGlo Reagent (Promega).

BiTE assay. 25,000 Target cells/well were seeded on a 96-well plate. The next day, 50,000 Jurkat NFAT-GFP reporter cells/well and BiTE were added. After 20 hours, Jurkat cells were recovered by gentle pipetting and washed in PBS plus 3\% BSA; GFP expression was quantified by flow cytometry using a CytoFLEX (Beckman Coulter) flow cytometer.

${ }^{89} \mathrm{Zr}$-IgG PET/CT mouse imaging studies. $1 \mathrm{mg}$ of IgG was mixed with $0.1 \mathrm{M}$ sodium bicarbonate $\mathrm{pH}$ 9.0. p-Isothiocyanatobenzyl-desferrioxamine (Df-Bz-NCS) was added at 3-fold molar excess. After 60 minutes at $37^{\circ} \mathrm{C}$, the reaction mixture was purified into $\mathrm{PBS} \mathrm{pH} 7.4$ via a G-25 column. In a reaction vial, ${ }^{89} \mathrm{Zr}$-oxalic acid solution $(5 \mathrm{mCi} ; 10$ $\mu \mathrm{L}$ ) was neutralized with $200 \mu \mathrm{L}$ of $1 \mathrm{M}$ HEPES pH 7.4, and $0.5 \mathrm{mg}$ of IgG-DFO was added to the reaction vial. After 60 minutes at $37^{\circ} \mathrm{C}$, radiolabeling efficiency was determined by instant thin layer chromatography (ITLC) using $20 \mathrm{mM}$ citric acid (pH 4.9-5.1). The radiolabeling efficiency was consistently greater than $98.5 \%$. Purification and buffer exchange into PBS were performed using a G-25 column. ${ }^{89} \mathrm{Zr}$-IgG was further diluted with $0.9 \%$ sodium chloride injection, USP, before i.v. administration for PET imaging.

Four- to 6-week-old intact male athymic nu/nu mice (Charles River Laboratory) bearing subcutaneous PL5 tumors were used for IgG
CL03 PET imaging. Six- to eight-week-old healthy immunocompetent male black 6 (C57BL/6J) mice (Jackson Laboratory) bearing subcutaneous Fc1245 c-CDCP1 tumors were used for IgG58 or IgG12 PET imaging. Tumor-bearing mice received $200 \mu \mathrm{Ci}$ of labeled Abs in 100 $\mu \mathrm{L}$ saline using a custom mouse tail-vein catheter. After 48 hours, mice were anesthetized with isoflurane and imaged on a small-animal PET/ CT scanner (Inveon, Siemens Healthcare). Decay corrected images were analyzed using AMIDE software, version 1.0.5. Forty-eight hours after radiotracer injection, animals were euthanized by cervical dislocation. Blood was harvested via cardiac puncture. Tissues were removed, weighed, and counted on a Hidex Automatic Gamma Counter for accumulation activity. The mass of the injected radiotracer was measured and used to determine the total cpm by comparison with a standard of known activity. The data were background and decay corrected and presented as the percentage of the injected dose/ weight of the biospecimen in grams (\% ID $/ \mathrm{g})$.

Mouse ADC toxicity study. The ADC labeling protocol was adapted from previous reports (55). The ADC toxicity study was performed with 8- to 10-week-old healthy male black 6(C57BL/6J) mice (Jackson Laboratory). Mice ( $n=5$ per group) were dosed i.v. weekly for 3 weeks with ADC $(15,10,5 \mathrm{mg} / \mathrm{kg}$ in $200 \mu \mathrm{L}$ PBS). Body weight was monitored biweekly for 4 weeks. Mice were euthanized if body weight dropped below $80 \%$.

${ }^{177} \mathrm{Lu}$-IgG mouse study. $2 \mathrm{mg}$ of IgG58 was mixed with $0.1 \mathrm{M}$ sodium bicarbonate $\mathrm{pH}$ 9.0. S-2-(4-isothiocyanatobenzyl)-1,4,7,10-tetraazacyclododecane tetraacetic acid (p-SCN-Bn-DOTA) was added at 50-fold molar excess. After 90 minutes at $37^{\circ} \mathrm{C}$, the IgG58-DOTA was purified via a G-25 column preequilibrated with $0.2 \mathrm{M}$ ammonium acetate $\mathrm{pH}$ 7.0. In a reaction vial, ${ }^{177} \mathrm{Lu}$-chloride solution $(6 \mathrm{mCi} ; 3 \mu \mathrm{L})$ was added directly to IgG58-DOTA. After incubation at $37^{\circ} \mathrm{C}$ for 60 minutes, radiolabeling efficiency was determined by ITLC. Radiolabeling efficiency was consistently greater than $98.5 \%$. Purification and buffer exchange into PBS was performed with a PD-10 column. The purified ${ }^{177} \mathrm{Lu}-\mathrm{IgG} 58$ was further diluted with $0.9 \%$ sodium chloride injection, USP, before being administered into mice.

Four- to six-week-old healthy male black 6 (C57BL/6J) mice (Jackson Laboratory) were inoculated s.c. with Fc1245 c-CDCP1 $(\sim 1.0 \times$ $10^{6}$ cells) in a 1:1 mixture (v/v) of media (DMEM) and Matrigel (Corning). Treatments were started 3 days after tumor implantation. Mice received ${ }^{177} \mathrm{Lu}-\mathrm{IgG} 58$ or vehicle (saline) at the indicated dose via tailvein injection. Mice were weighed and tumor size measured 3 times a week until the completion of the study. End points were euthanasia due to tumor volume of greater than $2000 \mathrm{~mm}^{3}$, tumor ulceration, or greater than $20 \%$ loss in body weight.

Statistics. All graphing and statistical analysis was performed in GraphPad Prism (version 8.4.2). For cell adhesion assay, unpaired, 2 -tailed $t$ test was used. For the ADC toxicity study, statistical analysis was performed using 1-way ANOVA. For the mouse efficacy study, unpaired, 2-tailed $t$ test was used. $P$ values of less than 0.05 were considered statistically significant.

Study approval. All mouse studies were conducted in compliance with and were approved by the IACUC at UCSF.

\section{Author contributions}

SAL and JZ designed and conducted all experiments unless otherwise noted. AJM conducted the characterization of PreScission-cleavable CDCP1. YHW and MJE conducted or supervised 
the PET imaging experiments. EVF and AAK conducted or supervised EM data collection and analysis. VS, DW, and $\mathrm{BH}$ conducted or supervised the mouse experiments. SGR prepared and analyzed the SAXS experiments. JL conducted the differential scanning fluorimetry experiments. KKL and JAW supervised the research. SAL, JZ, and JAW prepared and wrote the manuscript with input from all authors. SAL and JZ are co-first authors and contributed equally to the study. Their authorship order was chosen as alphabetical order by last name.

\section{Acknowledgments}

We thank members of the Wells Lab for helpful discussion and input and members of the Recombinant Antibody Network for feedback on this project. We would also like to thank Dan Zhu, Hari Hariharan, Henry Chan, and Ho Cho of Bristol Myers Squibb for their insights into this work. We also thank Shalini Chopra, Nima Hooshdaran, Fernando Salangsang, and Paul Phojanakong for assistance with the in vivo mouse studies. We also thank Susan Marqusee (UC Berkeley) for access to a CD spectrophotometer and Michal Hammel and Daniel Rosenberg of BL12.3.1 for their assistance in data collection and initial analysis. SAL is a Merck Fellow of the Helen Hay Whitney Foundation. JZ is supported by an NIH National Cancer Institute grant (F32 5F32CA236151). MJE was supported by the Department of Defense (PC200407), a Challenge Award from the Prostate Cancer Foundation, and the National Institute of Biomedical Imaging and Bioengineering (R01EB025207). JAW acknowledges funding from NIH NCI 1P41CA196276, CA191018, and NIH GM097316 and commercial funding from Bristol Myers Squibb. SAXS data were collected at BL12.3.1 at the Advanced Light Source (ALS), a national user facility operated by Lawrence Berkeley National Laboratory on behalf of the Department of Energy, Office of Basic Energy Sciences, through the Integrated Diffraction Analysis Technologies (IDAT) program, supported by DOE Office of Biological and Environmental Research. Additional support comes from the NIH project ALS-ENABLE (P30 GM124169) and a High-End Instrumentation grant (S10OD018483).

Address correspondence to: James A. Wells, UCSF Byers Hall Box 2552, 1700 4th Street, Room 504, San Francisco, California 94143, USA. Phone: 415.514.4498; Email: jim.wells@ucsf.edu.

SAM's present address is: Department of Antibody Engineering, Genentech Inc., South San Francisco, California, USA.

AJM's present address is: Soteria Biotherapeutics, San Francisco, California, USA.

JL's present address is: Merck \& Co., South San Francisco, California, USA.
1. Douglass J, et al. Bispecific antibodies targeting mutant $R A S$ neoantigens. Sci Immunol. 2021;6(57):eabd5515.

2. Hsiue EHC, et al. Targeting a neoantigen derived from a common TP53 mutation. Science. 2021;371(6533):eabc8697.

3. Blobel CP. Remarkable roles of proteolysis on and beyond the cell surface. Curr Opin Cell Biol. 2000;12(5):606-612.

4. Hanahan D, Weinberg RA. Hallmarks of cancer: the next generation. Cell. 2011;144(5):646-674.

5 . Sevenich L, Joyce JA. Pericellular proteolysis in cancer. Genes Dev. 2014;28(21):2331-2347.

6. Weeks AM, et al. Mapping proteolytic neo-N termini at the surface of living cells. Proc Natl Acad Sci U S A. 2021;118(8):e2018809118.

7. Mahrus S, et al. Global sequencing of proteolytic cleavage sites in apoptosis by specific labeling of protein N termini. Cell. 2008;134(5):866-876.

8. Desnoyers LR, et al. Tumor-specific activation of an EGFR-targeting probody enhances therapeutic index. Sci Transl Med. 2013;5(207):207ra144.

9. Dennis MS, et al. Peptide exosite inhibitors of factor VIIa as anticoagulants. Nature. 2000;404(6777):465-470.

10. Ai X, et al. Generation and characterization of antibodies specific for caspase-cleaved neo-epitopes: a novel approach. Cell Death Dis. 2011;2(9):1-8

11. Pylayeva-Gupta Y, et al. RAS oncogenes: weaving a tumorigenicweb. Nat Rev Cancer. 2011;11(11):761-774.

12. Wortmann A, et al. The cell surface glycoprotein CDCP1 in cancer - insights, opportunities, and challenges. IUBMB Life. 2009;61(7):723-730.

13. Scherl-Mostageer M, et al. Identification of a novel gene, CDCP1, overexpressed in human colorectal cancer. Oncogene. 2001;20(32):4402-4408.

14. Hooper JD, et al. Subtractive immunization using highly metastatic human tumor cells identifies SIMA135/CDCP1, a 135 kDa cell surface phosphorylated glycoprotein antigen. Oncogene. 2003;22(12):1783-1794.

15. Martinko AJ, et al. Targeting RAS-driven human cancer cells with antibodies to upregulated and essential cell-surface proteins. Elife. 2018;7:e31098.

16. Moroz A, et al. Theranostic targeting of CUB domain containing protein 1 (CDCP1) in pancreatic cancer. Clin Cancer Res. 2020;26(14):3608-3615.

17. Miyazawa Y, et al. CUB domain-containing protein 1, a prognostic factor for human pancreatic cancers, promotes cell migration and extracellular matrix degradation. Cancer Res. 2010;70(12):5136-5146

18. Uekita T, Sakai R. Roles of CUB domain-containing protein 1 signaling in cancer invasion and metastasis. Cancer Sci. 2011;102(11):1943-1948.

19. Casar B, et al. In vivo cleaved CDCP1 promotes early tumor dissemination via complexing with activated $\beta 1$ integrin and induction of FAK/PI3K/Akt motility signaling. Oncogene. 2014;33(2):255-268.

20. He Y, et al. Elevated CDCP1 predicts poor patient outcome and mediates ovarian clear cell carcinoma by promoting tumor spheroid formation, cell migration and chemoresistance. Oncogene. 2016;35(4):468-478.

21. Uekita T, et al. Oncogenic Ras/ERK signaling activates CDCP1 to promote tumor invasion and metastasis. Mol Cancer Res. 2014;12(10):1449-1459.
22. Wright HJ, et al. CDCP1 drives triple-negative breast cancer metastasis through reduction of lipid-droplet abundance and stimulation of fatty acid oxidation. Proc Natl Acad Sci U S A. 2017;114(32):E6556-E6565.

23. Wright HJ, et al. CDCP1 cleavage is necessary for homodimerization-induced migration of triple-negative breast cancer. Oncogene. 2016;35(36):4762-4772.

24. He Y, et al. Proteolysis-induced N-terminal ectodomain shedding of the integral membrane glycoprotein CUB domain-containing protein 1 (CDCP1) is accompanied by tyrosine phosphorylation of its $\mathrm{C}$-terminal domain and recruitment of Src and PKCdelta. J Biol Chem. 2010;285(34):26162-26173.

25. Alvares SM, et al. The role of membrane microdomains in transmembrane signaling through the epithelial glycoprotein Gp140/CDCP1. Biochim Biophys Acta. 2008;1780(3):486-496.

26. McGovern JA, et al. Stratum basale keratinocyte expression of the cell-surface glycoprotein CDCP1 during epidermogenesis and its role in keratinocyte migration. Br J Dermatol. 2013;168(3):496-503.

27. Wong CH, et al. Phosphorylation of the SRC epithelial substrate Trask is tightly regulated in normal epithelia but widespread in many human epithelial cancers. Clin Cancer Res. 2009;15(7):2311-2322.

28. Kryza T, et al. Substrate-biased activity-based probes identify proteases that cleave receptor CDCP1. Nat Chem Biol. 2021;17(7):776-783.

29. Jumper J, et al. Highly accurate protein structure prediction with AlphaFold. Nature. 2021;596(7873):583-589. 
30. Leroy C, et al. CUB-domain-containing protein 1 overexpression in solid cancers promotes cancer cell growth by activating Src family kinases. Oncogene. 2015;34(44):5593-5598.

31. Hornsby M, et al. A high through-put platform for recombinant antibodies to folded proteins. $\mathrm{Mol}$ Cell Proteomics. 2015;14(10):2833-2847.

32. Deryugina EI, et al. Functional role of cell surface CUB domain-containing protein 1 in tumor cell dissemination. Mol Cancer Res. 2009;7(8):1197-1211.

33. Ereño-Orbea J, et al. Structural basis of enhanced crystallizability induced by a molecular chaperone for antibody antigen-binding fragments. J Mol Biol. 2018;430(3):322-336.

34. Roy I, et al. Pancreatic cancer cell migration and metastasis is regulated by chemokine-biased agnoism and bioenergetic signaling. Cancer Res. 2015;75(17):3529-3542.

35. Ikeda JI, et al. Expression of CUB domain containing protein (CDCP1) is correlated with prognosis and survival of patients with adenocarcinoma of lung. Cancer Sci. 2009;100(3):429-433.

36. Chiu K, et al. ADAM 9 enhances CDCP1 by inhibiting miR-1 through EGFR signaling activation in lung cancer metastasis. Oncotarget. 2017;8(29):47365-47378.

37. Forte $L$, et al. The PDGFR $\beta / E R K 1 / 2$ pathway regulates $\mathrm{CDCP} 1$ expression in triple-negative breast cancer. BMC Cancer. 2018;18(1):1-11.

38. Uekita $\mathrm{T}$, et al. Novel small molecule inhibiting CDCP1-PKCd pathway reduces tumor metastasis and proliferation. Cancer Sci. 2017;108(5):1049-1057.

39. Fukuchi K, et al. Inhibition of tumor metastasis: functional immune modulation of the CUB domain containing protein 1. Mol Pharm. 2010;7(1):245-253.

40. Kollmorgen G, et al. Antibody mediated CDCP1 degradation as mode of action for cancer targeted therapy. Mol Oncol. 2013;7(6):1142-1151.

41. Siva AC, et al. Targeting CUB domain-containing protein 1 with a monoclonal antibody inhibits metastasis in a prostate cancer model. Cancer Res. 2008;68(10):3759-3766.

42. Spassov DS, et al. The transmembrane src substrate Trask is an epithelial protein that signals during anchorage deprivation. Am J Pathol. 2009;174(5):1756-1765.

43. Spassov DS, et al. Trask loss enhances tumorigenic growth by liberating integrin sigaling and growth factor receptor cross-talk in unanchored cells. Cancer Res. 2013;73(3):1168-1179.

44. Kryza T, et al. Effective targeting of intact and proteolysed CDCP1 for imaging and treatment of pancreatic ductal adenocarcinoma. Theranostics. 2020;10(9):4116-4133.

45. Bhatt AS, et al. Adhesion signaling by a novel mitotic substrate of src kinases. Oncogene. 2005;24(34):5333-5343.

46. Paciucci R, et al. The plasminogen activator system in pancreas cancer: role of t-PA in the invasive potential in vitro. Oncogene. 1998;16(5):625-633. 47. McMahon BJ, Kwaan HC. Components of the plasminogen-plasmin system as biologic markers for cancer. Adv Exp Med Biol. 2015;867:145-156

48. Tahmasebi S, et al. Solid tumors challenges and new insights of CAR T cell engineering. Stem Cell Rev Rep. 2019;15(5):619-636.

49. Nixon NA, et al. Current landscape of immunotherapy in the treatment of solid tumours, with future opportunities and challenges. Curr Oncol. 2018;25(5):e373-e384.

50. Dyer K, et al. High-throughput SAXS for the characterization of biomolecules in solution: a practical approach. Methods Mol Biol. 2014;1091:245-258.

51. König S, et al. Synchrotron radiation solution $\mathrm{X}$-ray scattering study of the $\mathrm{pH}$ dependence of the quaternary structure of yeast pyruvate decarboxylase. Biochemistry. 1992;31(37):8726-8731.

52. Scheres SHW. Amyloid structure determination in RELION-3.1. Acta Crystallogr D Struct Biol. 2020;D76(pt 2):94-101.

53. Pettersen EF, et al. UCSF Chimera--a visualization system for exploratory research and analysis. JComput Chem. 2004;25(13):1605-1612.

54. Elledge SK, et al. Systematic identification of engineered methionines and oxaziridines for efficient, stable, and site-specific antibody bioconjugation. Proc Natl Acad Sci U S A. 2020;117(11):5733-5740.

55. Doronina SO, et al. Development of potent monoclonal antibody auristatin conjugates for cancer therapy Corrigendum: A model of molecular interactions on short oligonucleotide microarrays. Nat Biotech. 2003;21(8):2003. 